\title{
THE KDV HIERARCHY AND ASSOCIATED TRACE FORMULAS
}

\author{
F. GESZTESY, R. RATNASEELAN, AND G. TESCHL
}

\begin{abstract}
A natural algebraic approach to the KdV hierarchy and its algebro-geometric finite-gap solutions is developed. In addition, a new derivation of associated higher-order trace formulas in connection with one-dimensional Schrödinger operators is presented.
\end{abstract}

\section{INTRODUCTION}

The purpose of this paper is to advocate a most natural algebraic approach to hierarchies of completely integrable evolution equations such as the AKNS and Toda hierarchies and a systematic treatment of associated trace formulas. Specifically, we shall treat in great detail the simplest example of these completely integrable systems, the Korteweg-de Vries (KdV) hierarchy, and derive the corresponding higher-order trace formulas for onedimensional Schrödinger operators. Even though the main ingredients of our approach to the KdV hierarchy (to be outlined below) appear to be well-known, it seems to us that no systematic attempt to combine them all into a complete description of the KdV hierarchy and its algebro-geometric solutions has been undertaken in the literature thus far. The principal aim of this paper is to fill this gap and at the same time provide the intimate connection with general higher-order trace formulas for the associated Lax operator.

The key ingredients just mentioned are a recursive approach to Lax pairs following Al'ber [1], 2] (see also [9], Ch. 12, [15]), naturally leading to the celebrated Burchnall-Chaundy polynomial [6], [7] and hence to hyperelliptic curves $K_{g}$ of genus $g \in \mathbb{N}_{0}(=\mathbb{N} \cup\{0\})$ and a classic representation of positive divisors of $K_{g}$ of degree $g$ due to Jacobi [27] and first applied to the KdV case by Mumford [36], Section III a).1, with subsequent extensions due to McKean [33]. Finally, following a recent series of papers on trace formulas for Schrödinger operators [16][19], 22]-24] we present a new algorithm for deriving higher-order trace formulas associated with the KdV hierarchy.

In Section 2 we briefly review Al'ber's recursive approach to the KdV hierarchy. In particular, we illustrate the role of commuting differential expressions of order $2 g+1, g \in \mathbb{N}_{0}$ and 2, respectively, in connection with the Burchnall-Chaundy polynomial, hyperelliptic curves $K_{g}$ of genus $g$ branched at infinity, and the equations of the stationary (i.e., timeindependent) KdV hierarchy. Section 3 combines Al'ber's recursion formalism with Jacobi's representation of positive divisors of degree $g$ of $K_{g}$ as applied to the KdV case by Mumford and McKean and provides a detailed construction of the stationary KdV hierarchy and its algebro-geometric solutions. The principal new result of Section 3, summarized in (3.50)(3.64), concern divisors of degree $g+1$ of $K_{g}$ associated with Schrödinger-type operators with

1991 Mathematics Subject Classification. Primary 35Q53, 34B05; Secondary 34B27, 35Q51.

Key words and phrases. KdV hierarchy, trace formulas.

in Recent Developments in Operator Theory and its Applications, I. Gohberg (ed.) et al., 125-163, Oper. Theory Adv. Appl. 87, Birkhäuser, Basel, 1996. 
general boundary conditions of the type defined in (3.45). In Section 4 we present a systematic extension of this body of ideas to the time-dependent KdV hierarchy going beyond the standard treatment in the literature. Especially, our $t$-dependent discussion in connection with divisors of degree $g+1$ of $K_{g}$ associated with the general eigenvalue problem (3.45) as presented in 4.36- 4.50 is without precedent. Moreover, our proof of the theta function representation (4.51) of the Baker-Akhiezer function $\psi\left(P, x, x_{0}, t, t_{0}\right)$ in Theorem 4.6, based on the fundamental meromorphic function $\phi(P, x, t)$ defined in (4.15), is new. In Section 5 we turn to (higher-order) trace formulas for Schrödinger operators associated with general boundary conditions (cf. (5.3)), a key ingredient in the solution of inverse spectral problems. Unlike Sections 3 and 4, the approach in Section 5 applies to general (not necessarily algebrogeometric finite-gap) solutions of the KdV hierarchy. The principal new results of Section 5 are the (universally valid) nonlinear differential equation $(5.18)$ for $\Gamma^{\beta}(z, x), \beta \in \mathbb{R}$ (defined in (5.4)), the resulting recursion relation (5.21), and, in particular, our method of proof of Theorem 5.3 (i). In Appendix A we provide a brief summary on hyperelliptic curves of the $\mathrm{KdV}$-type and their theta functions and establish our basic notation used in Sections 3 and 4. Finally, Appendix B provides an explicit illustration of the Riemann-Roch theorem in connection with hyperelliptic curves branched at infinity which appears to be of independent interest.

We emphasize that the methods of this paper are widely applicable to $1+1$-dimensional completely integrable systems. The corresponding account for the Toda and Kac-van Moerbeke hierarchy can be found in [5].

\section{The KdV Hierarchy, Recursion Relations, and Hyperelliptic Curves}

In this section we briefly review the construction of the KdV hierarchy using a recursive approach advocated by Al'ber [1], 2] (see also [9], Ch. 12, [15], 20]) and outline its connection with the Burchnall-Chaundy polynomial [6], [7] and associated hyperelliptic curves branched at infinity.

Suppose

$$
V(., t) \in C^{\infty}(\mathbb{R}), t \in \mathbb{R}, V(x, .) \in C^{1}(\mathbb{R}), x \in \mathbb{R}
$$

and consider the differential expressions (Lax pair)

$$
\begin{gathered}
L(t)=-\frac{d^{2}}{d x^{2}}+V(x, t), \\
P_{2 g+1}(t)=\sum_{j=0}^{g}\left[f_{j}(x, t) \frac{d}{d x}-\frac{1}{2} f_{j, x}(x, t)\right] L(t)^{g-j}, \quad g \in \mathbb{N}_{0},(x, t) \in \mathbb{R}^{2},
\end{gathered}
$$

where the $\left\{f_{j}\right\}_{0 \leq j \leq g}$ satisfy the recursion relation

$$
f_{0}=1, f_{j, x}=-\frac{1}{4} f_{j-1, x x x}+V f_{j-1, x}+\frac{1}{2} V_{x} f_{j-1}, \quad 1 \leq j \leq g .
$$

Define in addition $f_{g+1}$ by

$$
f_{g+1, x}=-\frac{1}{4} f_{g, x x x}+V f_{g, x}+\frac{1}{2} V_{x} f_{g}
$$

Then one computes

$$
\left[P_{2 g+1}, L\right]=2 f_{g+1, x}
$$


where [.,.] denotes the commutator. The Lax equation

$$
\frac{d}{d t} L(t)-\left[P_{2 g+1}(t), L(t)\right]=0, \quad t \in \mathbb{R}
$$

is then equivalent to

$$
\mathrm{KdV}_{g}(V)=V_{t}-2 f_{g+1, x}, \quad t \in \mathbb{R} .
$$

Varying $g \in \mathbb{N}_{0}$ yields the KdV hierarchy

$$
\operatorname{KdV}_{g}(V)=0, \quad g \in \mathbb{N}_{0} .
$$

Explicitly, one obtains from (2.4),

$$
\begin{aligned}
f_{0} & =1=\tilde{f}_{0}, \\
f_{1} & =\frac{1}{2} V+c_{1}=c_{1} \tilde{f}_{0}+\tilde{f}_{1} \\
f_{2} & =-\frac{1}{8} V_{x x}+\frac{3}{8} V^{2}+c_{1} \frac{1}{2} V+c_{2}=c_{2} \tilde{f}_{0}+c_{1} \tilde{f}_{1}+\tilde{f}_{2}, \\
f_{3} & =\frac{1}{32} V_{x x x x}-\frac{5}{16} V V_{x x}-\frac{5}{32} V_{x}^{2}+\frac{5}{16} V^{3}+c_{2} \frac{1}{2} V+c_{1}\left[-\frac{1}{8} V_{x x}+\frac{3}{8} V^{2}\right]+c_{3} \\
& =c_{3} \tilde{f}_{0}+c_{2} \tilde{f}_{1}+c_{1} \tilde{f}_{2}+\tilde{f}_{3},
\end{aligned}
$$

etc.

Hence by 2.8,

$$
\begin{aligned}
\mathrm{KdV}_{0}(V) & =V_{t}-V_{x}=0 \\
\mathrm{KdV}_{1}(V) & =V_{t}+\frac{1}{4} V_{x x x}-\frac{3}{2} V V_{x}-c_{1} V_{x} \\
\mathrm{KdV}_{2}(V) & =V_{t}-\frac{1}{16} V_{x x x x x}+\frac{5}{8} V V_{x x x}+\frac{5}{4} V_{x} V_{x x}-\frac{15}{8} V^{2} V_{x}-c_{2} V_{x}+c_{1}\left[\frac{1}{4} V_{x x x}-\frac{3}{2} V V_{x}\right], \\
& \text { etc. }
\end{aligned}
$$

represent the first few equations of the KdV hierarchy. Here $c_{\ell}$ denote integration constants which naturally arise when solving (2.4). Moreover, the corresponding homogeneous $\mathrm{KdV}$ equations, obtained by taking all integration constants equal to zero, $c_{\ell} \equiv 0, \ell \geq 1$ are then denoted by

$$
\widetilde{\operatorname{KdV}_{g}}(V):=\left.\operatorname{KdV}_{g}(V)\right|_{c_{\ell} \equiv 0,1 \leq \ell \leq g}
$$

and similarly we denote by $\tilde{P}_{2 g+1}:=P_{2 g+1}\left(c_{\ell} \equiv 0\right), \tilde{f}_{j}:=f_{j}\left(c_{\ell} \equiv 0\right)$, etc. the corresponding homogeneous quantities.

Before we turn to a discussion of the stationary KdV hierarchy we briefly sketch the main steps leading to (2.3)-(2.8). Let $\operatorname{Ker}(L(t)-z), z \in \mathbb{C}$ denote the two-dimensional nullspace of $L(t)-z$ (in the algebraic sense as opposed to the functional analytic one). We seek a representation of $P_{2 g+1}(t)$ on $\operatorname{Ker}(L(t)-z)$ of the form

$$
\left.P_{2 g+1}(t)\right|_{\operatorname{Ker}(L(t)-z)}=F_{g}(z, x, t) \frac{d}{d x}+G_{g-1}(z, x, t),
$$

where $F_{g}$ are polynomials in $z$ of the type

$$
\begin{aligned}
F_{g}(z, x, t) & =\sum_{j=0}^{g} f_{g-j}(x, t) z^{j}, \\
G_{g-1}(z, x, t) & =\sum_{j=0}^{g-1} g_{g-j}(x, t) z^{j} .
\end{aligned}
$$


The Lax equation (2.7) restricted to $\operatorname{Ker}(L(t)-z)$ then yields

$$
\begin{aligned}
0 & =\left.\left\{\dot{L}-\left[P_{2 g+1}, L\right]\right\}\right|_{\operatorname{Ker}(L-z)}=\left.\left\{\dot{L}+(L-z) P_{2 g+1}\right\}\right|_{\operatorname{Ker}(L-z)} \\
& =\left.\left\{-\left[F_{g, x x}+2 G_{g-1, x}\right] \frac{d}{d x}+\left[V_{t}-F_{g} V_{x}-2(V-z) F_{g, x}-G_{g-1, x x}\right]\right\}\right|_{\operatorname{Ker}(L-z)}
\end{aligned}
$$

implying

$$
G_{g-1}=-F_{g, x} / 2
$$

(neglecting a trivial integration constant) and

$$
V_{t}=-\frac{1}{2} F_{g, x x x}+2(V-z) F_{g, x}+V_{x} F_{g} .
$$

Insertion of (2.14) into (2.18) then yields (2.8). We omit further details and just record a few of the polynomials $F_{g}$,

$$
\begin{aligned}
& F_{0}=1=\tilde{F}_{0} \\
& F_{1}=c_{1}+\frac{1}{2} V+z=c_{1} \tilde{F}_{0}+\tilde{F}_{1}, \\
& F_{2}=c_{2}+c_{1} \frac{1}{2} V-\frac{1}{8} V_{x x}+\frac{3}{8} V^{2}+\left(c_{1}+\frac{1}{2} V\right) z+z^{2}=c_{2} \tilde{F}_{0}+c_{1} \tilde{F}_{1}+\tilde{F}_{2}, \\
& \quad \text { etc. }
\end{aligned}
$$

One verifies

$$
P_{2 g+1}=\sum_{m=0}^{g} c_{g-m} \tilde{P}_{2 m+1}, \quad c_{0}=1 .
$$

Finally, we specialize to the stationary KdV hierarchy characterized by $V_{t}=0$ in 2.9 ) (respectively (2.8)), or more precisely, by commuting differential expressions

$$
\left[P_{2 g+1}, L\right]=0
$$

of order $2 g+1$ and 2, respectively. Eq. (2.18) then becomes

$$
F_{g, x x x}-4(V-z) F_{g, x}-2 V_{x} F_{g}=0
$$

and upon multiplying by $F_{g}$ and integrating one infers

$$
\frac{1}{2} F_{g, x x} F_{g}-\frac{1}{4} F_{g, x}^{2}-(V-z) F_{g}^{2}=R_{2 g+1}(z),
$$

where $R_{2 g+1}(z)$ is of the form

$$
R_{2 g+1}(z)=\prod_{n=0}^{2 g}\left(z-E_{n}\right), \quad\left\{E_{n}\right\}_{0 \leq n \leq 2 g} \subset \mathbb{C} .
$$

Because of 2.21) one computes

$$
\left[\left.P_{2 g+1}\right|_{\operatorname{Ker}(L-z)}\right]^{2}=-\left.\left[\frac{1}{2} F_{g, x x} F_{g}-\frac{1}{4} F_{g, x}^{2}-(V-z) F_{g}^{2}\right]\right|_{\operatorname{Ker}(L-z)}=-R_{2 g+1}(z) .
$$

Since $z \in \mathbb{C}$ is arbitrary, one obtains the Burchnall-Chaundy polynomial [6], 7] relating $P_{2 g+1}$ and $L$,

$$
-P_{2 g+1}^{2}=R_{2 g+1}(L)=\prod_{n=0}^{2 g}\left(L-E_{n}\right) .
$$


The resulting hyperelliptic curve $K_{g}$ of (arithmetic) genus $g$, obtained upon one-point compactification of the curve

$$
y^{2}=R_{2 g+1}(z)=\prod_{n=0}^{2 g}\left(z-E_{n}\right)
$$

(cf. Appendix A), will be the basic ingredient in our algebro-geometric treatment of the KdV hierarchy in Sections 3 and 4.

The spectral theoretic content of the polynomials $F_{g}, G_{g-1}$ is clearly displayed in 3.35), (3.37), 3.40) 3.44.

\section{The Stationary Formalism}

Combining the recursion formalism of Section 2 with a polynomial approach to represent positive divisors of degree $g$ of a hyperelliptic curve $K_{g}$ of genus $g$ originally developed by Jacobi [27] and applied to the KdV case by Mumford [36], Section III a).1 and McKean [33, we provide a detailed construction of the stationary KdV hierarchy and its algebrogeometric solutions. Our considerations (3.50)-3.64 in connection with the general $\beta$ boundary conditions for Schrödinger-type operators in (3.45) are new.

As indicated at the end of Section 2, the stationary KdV hierarchy is intimately connected with pairs of commuting differential expressions $P_{2 g+1}$ and $L$ of orders $2 g+1$ and 2 , respectively and hyperelliptic curves $K_{g}$ obtained upon one-point compactification of the curve

$$
y^{2}=R_{2 g+1}(z)=\prod_{n=0}^{2 g}\left(z-E_{n}\right)
$$

described in detail in Appendix A (whose results and notations we shall freely use in the remainder of this paper). Since we are interested in real-valued KdV solutions we now make the additional assumption

$$
\left\{E_{n}\right\}_{0 \leq n \leq 2 g} \subset \mathbb{R}, E_{0}<E_{1}<\cdots<E_{2 g}, \quad g \in \mathbb{N}_{0} .
$$

Writing

$$
F_{g}(z, x)=\sum_{j=0}^{g} f_{g-j}(x) z^{j}=\prod_{j=1}^{g}\left[z-\mu_{j}(x)\right]
$$

and combining (2.23) and (3.3) yields

$$
\mu_{j}^{\prime}(x)^{2}=-4 R_{2 g+1}\left(\mu_{j}(x)\right) \prod_{\substack{k=1 \\ k \neq j}}^{g}\left[\mu_{j}(x)-\mu_{k}(x)\right]^{-2}, \quad 1 \leq j \leq g, x \in \mathbb{R} .
$$

Integrating the nonlinear first-order system (3.4) as a vector field on the (complex) manifold $K_{g} \times \cdots \times K_{g}=K_{g}^{g}$, its solution is well-defined as long as the $\mu$ 's do not collide. Since we focus on real-valued solutions $V$ of the KdV hierarchy, we may restrict the vector field to the

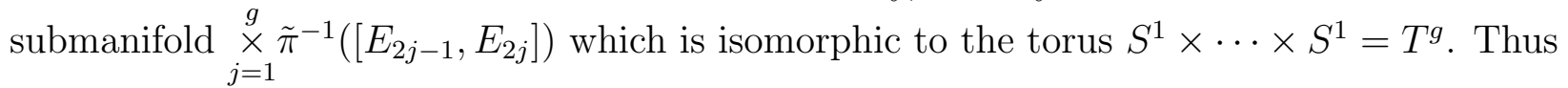

$$
\mu_{j}^{\prime}(x)=-2 i R_{2 g+1}^{1 / 2}\left(\hat{\mu}_{j}(x)\right) \prod_{\substack{k=1 \\ k \neq j}}^{g}\left[\mu_{j}(x)-\mu_{k}(x)\right]^{-1}, \quad 1 \leq j \leq g, x \in \mathbb{R},
$$


with the initial conditions

$$
\left\{\hat{\mu}_{j}\left(x_{0}\right)\right\}_{1 \leq j \leq g} \subset K_{g}, \tilde{\pi}\left(\hat{\mu}_{j}\left(x_{0}\right)\right)=\mu_{j}\left(x_{0}\right) \in\left[E_{2 j-1}, E_{2 j}\right], \quad 1 \leq j \leq g
$$

for some fixed $x_{0} \in \mathbb{R}$, has the unique solution $\left\{\hat{\mu}_{j}(x)\right\}_{1 \leq j \leq g} \subset K_{g}$ satisfying

$$
\hat{\mu}_{j}(.) \in C^{\infty}\left(\mathbb{R}, K_{g}\right), \tilde{\pi}\left(\hat{\mu}_{j}(x)\right) \in\left[E_{2 j-1}, E_{2 j}\right], \quad 1 \leq j \leq g, x \in \mathbb{R} .
$$

These facts are verified using the charts (A.7), A.8) which also shows that the solution $\hat{\mu}_{j}(x)$ changes sheets whenever it hits $E_{2 j-1}$ or $E_{2 j}$ and its projection $\mu_{j}(x)=\tilde{\pi}\left(\hat{\mu}_{j}(x)\right)$ remains trapped in $\left[E_{2 j-1}, E_{2 j}\right]$ for all $x \in \mathbb{R}$.

Given (3.3), (3.5), and 2.17) one obtains

$$
\begin{aligned}
G_{g-1}(z, x) & =-\frac{1}{2} F_{g, x}(z, x)=\frac{1}{2} \sum_{j=1}^{g} \mu_{j}^{\prime}(x) \prod_{\substack{k=1 \\
k \neq j}}^{g}\left[z-\mu_{k}(x)\right] \\
& =-i \sum_{j=1}^{g} R_{2 g+1}^{1 / 2}\left(\hat{\mu}_{j}(x)\right) \prod_{\substack{k=1 \\
k \neq j}}^{g}\left(\frac{z-\mu_{k}(x)}{\mu_{j}(x)-\mu_{k}(x)}\right)
\end{aligned}
$$

and hence

$$
\begin{aligned}
& R_{2 g+1}^{1 / 2}\left(\hat{\mu}_{j}(x)\right)=\sigma_{j}(x) R_{2 g+1}\left(\mu_{j}(x)\right)^{1 / 2}=i G_{g-1}\left(\mu_{j}(x), x\right) \\
& \hat{\mu}_{j}(x)=\left(\mu_{j}(x), i G_{g-1}\left(\mu_{j}(x), x\right)\right), \quad 1 \leq j \leq g
\end{aligned}
$$

Moreover, since

$$
\left.\left[R_{2 g+1}(z)+G_{g-1}(z, x)^{2}\right]\right|_{z=\mu_{j}(x)}=0, \quad 1 \leq j \leq g
$$

one infers

$$
R_{2 g+1}(z)+G_{g-1}(z, x)^{2}=F_{g}(z, x) H_{g+1}(z, x)
$$

for some polynomial $H_{g+1}$ in $z$ of degree $g+1$,

$$
H_{g+1}(z, x)=\prod_{\ell=0}^{g}\left[z-\nu_{\ell}(x)\right] .
$$

Eqs. 3.9), 3.11), and (3.12) suggest defining $\left\{\hat{\nu}_{\ell}(x)\right\}_{0 \leq \ell \leq g} \subset K_{g}$ by

$$
R_{2 g+1}^{1 / 2}\left(\hat{\nu}_{\ell}(x)\right)=-i G_{g-1}\left(\nu_{\ell}(x), x\right), \hat{\nu}_{\ell}(x)=\left(\nu_{\ell}(x),-i G_{g-1}\left(\nu_{\ell}(x), x\right)\right), \quad 0 \leq \ell \leq g .
$$

One verifies

$$
\nu_{0}(x) \leq E_{0}, \nu_{\ell}(x) \in\left[E_{2 \ell-1}, E_{2 \ell}\right], \quad 1 \leq \ell \leq g, x \in \mathbb{R}
$$

Next, we define the fundamental meromorphic function $\phi(P, x)$ on $K_{g}$,

$$
\begin{aligned}
\phi(P, x) & =\frac{i R_{2 g+1}^{1 / 2}(P)-G_{g-1}(\tilde{\pi}(P), x)}{F_{g}(\tilde{\pi}(P), x)}=\frac{i R_{2 g+1}^{1 / 2}(P)+\frac{1}{2} F_{g, x}(\tilde{\pi}(P), x)}{F_{g}(\tilde{\pi}(P), x)} \\
& =\frac{-H_{g+1}(\tilde{\pi}(P), x)}{i R_{2 g+1}^{1 / 2}(P)+G_{g-1}(\tilde{\pi}(P), x)}, P=\left(\tilde{\pi}(P), R_{2 g+1}^{1 / 2}(P)\right), x \in \mathbb{R},
\end{aligned}
$$

with divisor $(\phi(., x))$ given by

$$
(\phi(., x))=\mathcal{D}_{\hat{\nu}_{0}(x) \underline{\hat{\nu}}(x)}-\mathcal{D}_{P_{\infty} \underline{\hat{\mu}}(x)} .
$$

Here we abbreviated

$$
\underline{\hat{\nu}}(x)=\left(\hat{\nu}_{1}(x), \ldots, \hat{\nu}_{g}(x)\right), \underline{\hat{\mu}}(x)=\left(\hat{\mu}_{1}(x), \ldots, \hat{\mu}_{g}(x)\right) .
$$


Given $\phi(P, x)$ we define the stationary Baker-Akhiezer (BA) function $\psi\left(P, x, x_{0}\right)$, meromorphic on $K_{g} \backslash\left\{P_{\infty}\right\}$, by

$$
\psi\left(P, x, x_{0}\right)=\exp \left[\int_{x_{0}}^{x} d y \phi(P, y)\right], \quad\left(x, x_{0}\right) \in \mathbb{R}^{2} .
$$

Properties of $V(x), \phi(P, x)$, and $\psi\left(P, x, x_{0}\right)$ are summarized in the following

Lemma 3.1. Let $P=\left(z, \sigma R_{2 g+1}(z)^{1 / 2}\right)=\left(\tilde{\pi}(P), R_{2 g+1}^{1 / 2}(P)\right) \in K_{g} \backslash\left\{P_{\infty}\right\},\left(z, x, x_{0}\right) \in \mathbb{C} \times \mathbb{R}^{2}$. Then

(i). $\quad V(x)=E_{0}+\sum_{j=1}^{g}\left[E_{2 j-1}+E_{2 j}-2 \mu_{j}(x)\right]$.

(ii). $\phi(P, x)$ satisfies the Riccati-type equation

$$
\phi_{x}(P, x)+\phi(P, x)^{2}=V(x)-z .
$$

(iii). $\quad \psi\left(P, x, x_{0}\right)$ satisfies the Schrödinger equation

$$
-\psi_{x x}\left(P, x, x_{0}\right)+[V(x)-z] \psi\left(P, x, x_{0}\right)=0 .
$$

(iv). $\quad \phi(P, x) \phi\left(P^{*}, x\right)=H_{g+1}(z, x) / F_{g}(z, x)$.

(v). $\phi(P, x)+\phi\left(P^{*}, x\right)=-2 G_{g-1}(z, x) / F_{g}(z, x)=F_{g, x}(z, x) / F_{g}(z, x)$.

(vi). $\quad \phi(P, x)-\phi\left(P^{*}, x\right)=2 i R_{2 g+1}^{1 / 2}(P) / F_{g}(z, x)$.

(vii). $\quad \psi\left(P, x, x_{0}\right) \psi\left(P^{*}, x, x_{0}\right)=F_{g}(z, x) / F_{g}\left(z, x_{0}\right)$.

(viii). $\quad \psi_{x}\left(P, x, x_{0}\right) \psi_{x}\left(P^{*}, x, x_{0}\right)=H_{g+1}(z, x) / F_{g}\left(z, x_{0}\right)$.

(ix). $\quad \psi\left(P, x, x_{0}\right)=\left[F_{g}(z, x) / F_{g}\left(z, x_{0}\right)\right]^{1 / 2} \exp \left[i R_{2 g+1}^{1 / 2}(P) \int_{x_{0}}^{x} d y F_{g}(z, y)^{-1}\right]$.

Proof. (i). Insert (3.3) into (2.23) and compare the coefficient of $z^{2 g}$. (ii). Combine (2.17), (2.23), and (3.15). (iii). Follows from $\psi_{x x} / \psi=\phi_{x}+\phi^{2}=V-z$. (iv). Multiply the first and third expression in (3.15) replacing $P$ by $P^{*}$ in one of the two factors. (v), (vi) are clear from (3.15). (vii). Combine (3.18) and (3.23). (viii). Use (3.22), (3.25), and $\psi_{x}=\phi \psi$. (ix). Invoke (2.17), (3.15), and (3.18).

Eq. (3.19) represents a trace formula for the finite-gap potential $V(x)$. The method of proof of Lemma 3.1 (i) indicates that higher-order trace formulas associated with the KdV hierarchy can be obtained from (3.3) and (2.23) comparing powers of $z$. Since we shall derive trace formulas for general potentials in Section 5, we postpone the special case of finite-gap potentials at this point and refer to Example 5.5.

We also record

Lemma 3.2. Let $(z, x) \in \mathbb{C} \times \mathbb{R}$. Then

(i). $\quad H_{g+1}(z, x)=\frac{1}{2} F_{g, x x}(z, x)-[V(x)-z] F_{g}(z, x)$.

(ii). $\quad H_{g+1, x}(z, x)=-2[V(x)-z] G_{g-1}(z, x)$. 
Proof. (i). By (2.17), 2.23), and (3.11),

$$
\begin{aligned}
& -\frac{1}{2} F_{g, x x}=-\frac{1}{4} F_{g}^{-1} F_{g, x}^{2}-(V-z) F_{g}-F_{g}^{-1} R_{2 g+1} \\
& =-(V-z) F_{g}-F_{g}^{-1}\left(R_{2 g+1}+G_{g-1}^{2}\right)=-(V-z) F_{g}-H_{g+1} .
\end{aligned}
$$

(ii). By 2.17), 2.22), and (3.28,

$$
\begin{aligned}
& H_{g+1, x}=-G_{g-1, x x}-(V-z) F_{g, x}-V_{x} F_{g} \\
& =\frac{1}{2} F_{g, x x x}-(V-z) F_{g, x}-V_{x} F_{g}=(V-z) F_{g, x}=-2(V-z) G_{g-1} .
\end{aligned}
$$

Explicitly, one computes from (2.4), 2.14), and (3.28),

$$
\begin{aligned}
H_{1} & =\tilde{H}_{1}=-V+z, \\
H_{2} & =-c_{1} V+\frac{1}{4} V_{x x}-\frac{1}{2} V^{2}+\left(c_{1}-\frac{1}{2} V\right) z+z^{2}=c_{1} \tilde{H}_{1}+\tilde{H}_{2}, \\
H_{3} & =-c_{2} V+c_{1}\left(\frac{1}{4} V_{x x}-\frac{1}{2} V^{2}\right)-\frac{1}{16} V_{x x x x}+\frac{3}{8} V_{x}^{2}+\frac{1}{2} V V_{x x}-\frac{3}{8} V^{3} \\
& +\left[c_{2}-c_{1} \frac{1}{2} V+\frac{1}{8} V_{x x}-\frac{1}{8} V^{2}\right] z+\left[c_{1}-\frac{1}{2} V\right] z^{2}+z^{3}=c_{2} \tilde{H}_{1}+c_{1} \tilde{H}_{2}+\tilde{H}_{3},
\end{aligned}
$$

etc.

We also mention the following well-known result connecting Dirichlet and Neumann eigenvalues.

Lemma 3.3. [33] Suppose $\mu_{j}\left(x_{0}\right) \in\left\{E_{2 j-1}, E_{2 j}\right\}, 1 \leq j \leq g$. Then $\nu_{0}\left(x_{0}\right)=E_{0}, \nu_{j}\left(x_{0}\right) \in$ $\left\{E_{2 j-1}, E_{2 j}\right\} \backslash\left\{\mu_{j}\left(x_{0}\right)\right\}, 1 \leq j \leq g$. Conversely, suppose $\nu_{j}\left(x_{0}\right) \in\left\{E_{2 j-1}, E_{2 j}\right\}, 1 \leq j \leq g$. Then $\nu_{0}\left(x_{0}\right)=E_{0}, \mu_{j}\left(x_{0}\right) \in\left\{E_{2 j-1}, E_{2 j}\right\} \backslash\left\{\nu_{j}\left(x_{0}\right)\right\}, 1 \leq j \leq g$.

Proof. If $\mu_{j}\left(x_{0}\right) \in\left\{E_{2 j-1}, E_{2 j}\right\}, 1 \leq j \leq g$ then $G_{g-1}\left(z, x_{0}\right)=0$ in (3.11) yields $R_{2 g+1}(z)=$ $F_{g}\left(z, x_{0}\right) H_{g+1}\left(z, x_{0}\right)$ and hence proves the first claim. Conversely, assuming $\nu_{j}\left(x_{0}\right) \in\left\{E_{2 j-1}, E_{2 j}\right\}$, $1 \leq j \leq g$ one infers from (3.13) that $G_{g-1}\left(\nu_{j}\left(x_{0}\right), x_{0}\right)=i R_{2 g+1}^{1 / 2}\left(\hat{\nu}_{j}\left(x_{0}\right)\right)=0,1 \leq j \leq g$, i.e., again $G_{g-1}\left(z, x_{0}\right)=0$. Hence $R_{2 g+1}(z)=F_{g}\left(z, x_{0}\right) H_{g+1}\left(z, x_{0}\right)$ also proves the second claim.

Given the bounded potential $V(x)$ in 3.19 , consider the differential expression $\tau=-\frac{d^{2}}{d x^{2}}+$ $V(x)$ and define the corresponding self-adjoint Schrödinger operator $H$ in $L^{2}(\mathbb{R})$ by

$$
H f=\tau f, \tau=-\frac{d^{2}}{d x^{2}}+V(x), \quad x \in \mathbb{R}, f \in \mathcal{D}(H)=H^{2,2}(\mathbb{R}),
$$

with $H^{m, n}($.$) the usual Sobolev spaces. The resolvent of H$ reads

$$
\left((H-z)^{-1} f\right)(x)=\int_{\mathbb{R}} d x^{\prime} G\left(z, x, x^{\prime}\right) f\left(x^{\prime}\right), z \in \mathbb{C} \backslash \sigma(H), f \in L^{2}(\mathbb{R}),
$$

where the Green's function $G\left(z, x, x^{\prime}\right)$ is explicitly given by

$$
G\left(z, x, x^{\prime}\right)=W\left(\psi_{+}\left(z, ., x_{0}\right), \psi_{-}\left(z, ., x_{0}\right)\right)^{-1} \begin{cases}\psi_{+}\left(z, x, x_{0}\right) \psi_{-}\left(z, x^{\prime}, x_{0}\right), & x \geq x^{\prime} \\ \psi_{+}\left(z, x^{\prime}, x_{0}\right) \psi_{-}\left(z, x, x_{0}\right), & x \leq x^{\prime}\end{cases}
$$

with $W(f, g)=f g^{\prime}-f^{\prime} g$ the Wronskian of $f$ and $g$ and $\psi_{ \pm}\left(z, x, x_{0}\right)$ the branches of $\psi\left(P, x, x_{0}\right)$ in the charts $\left(\Pi_{ \pm}, \tilde{\pi}\right)$. One computes

$$
W\left(\psi_{+}\left(z, ., x_{0}\right), \psi_{-}\left(z, ., x_{0}\right)\right)=(2 / i) R_{2 g+1}(z)^{1 / 2} F_{g}\left(z, x_{0}\right)^{-1}
$$


and

$$
G(z, x, x)=\frac{i \prod_{j=1}^{g}\left[z-\mu_{j}(x)\right]}{2 R_{2 g+1}(z)^{1 / 2}}=\frac{i F_{g}(z, x)}{2 R_{2 g+1}(z)^{1 / 2}},
$$

taking into account our convention A.3 for $R_{2 g+1}(z)^{1 / 2}$. In particular, the spectrum $\sigma(H)$ of $H$ is given by

$$
\sigma(H)=\bigcup_{j=0}^{g-1}\left[E_{2 j}, E_{2 j+1}\right] \cup\left[E_{2 g}, \infty\right) .
$$

Eq. (3.35) illustrates the spectral theoretic content of the polynomial $F_{g}(z, x)$. Moreover, the Weyl $m$-functions $m_{ \pm}\left(z, x_{0}\right)$, associated with the restriction of $\tau$ to $\left(x_{0}, \pm \infty\right)$ with a Dirichlet boundary condition at $x_{0}$, read

$$
m_{ \pm}\left(z, x_{0}\right)=\phi_{ \pm}\left(z, x_{0}\right)=\left[ \pm i R_{2 g+1}(z)^{1 / 2}-G_{g-1}\left(z, x_{0}\right)\right] F_{g}\left(z, x_{0}\right)^{-1}
$$

where $\phi_{ \pm}(z, x)$ denote the branches of $\phi(P, x)$ in the charts $\left(\Pi_{ \pm}, \tilde{\pi}\right)$. As a consequence, the Weyl $m$-matrix $M\left(z, x_{0}\right)$ associated with $H$ is given by (see, e.g., [32], Ch. 8)

$$
\begin{aligned}
M\left(z, x_{0}\right) & =\left[m_{-}\left(z, x_{0}\right)-m_{+}\left(z, x_{0}\right)\right]^{-1}\left(\begin{array}{cc}
m_{-}\left(z, x_{0}\right) m_{+}\left(z, x_{0}\right) & {\left[m_{-}\left(z, x_{0}\right)+m_{+}\left(z, x_{0}\right)\right] / 2} \\
{\left[m_{-}\left(z, x_{0}\right)+m_{+}\left(z, x_{0}\right)\right] / 2} & 1
\end{array}\right) \\
& =\left(\begin{array}{cc}
\partial_{1} \partial_{2} G\left(z, x_{0}, x_{0}\right) & \frac{1}{2}\left(\partial_{1}+\partial_{2}\right) G\left(z, x_{0}, x_{0}\right) \\
\frac{1}{2}\left(\partial_{1}+\partial_{2}\right) G\left(z, x_{0}, x_{0}\right) & G\left(z, x_{0}, x_{0}\right)
\end{array}\right) \\
& =\frac{i}{2 R_{2 g+1}(z)^{1 / 2}}\left(\begin{array}{cc}
H_{g+1}\left(z, x_{0}\right) & -G_{g-1}\left(z, x_{0}\right) \\
-G_{g-1}\left(z, x_{0}\right) & F_{g}\left(z, x_{0}\right)
\end{array}\right),
\end{aligned}
$$

where

$$
\begin{aligned}
\partial_{1} G\left(z, x_{0}, x^{\prime}\right) & =\left.\partial_{x} G\left(z, x, x^{\prime}\right)\right|_{x=x_{0}}, \partial_{2} G\left(z, x, x_{0}\right)=\left.\partial_{x^{\prime}} G\left(z, x, x^{\prime}\right)\right|_{x^{\prime}=x_{0}}, \\
\partial_{1} \partial_{2} G\left(z, x_{0}, x_{0}\right) & =\left.\partial_{x} \partial_{x^{\prime}} G\left(z, x, x^{\prime}\right)\right|_{x=x_{0}=x^{\prime}}, \quad \text { etc. }
\end{aligned}
$$

The corresponding self-adjoint spectral matrix $\rho\left(\lambda, x_{0}\right)$, defined by

$$
\begin{aligned}
& M_{p, q}\left(z, x_{0}\right)=\int_{\mathbb{R}}(z-\lambda)^{-1} d \rho_{p, q}\left(\lambda, x_{0}\right), \\
& \rho_{p, q}\left(\lambda, x_{0}\right)-\rho_{p, q}\left(\mu, x_{0}\right)=\lim _{\delta \downarrow 0} \lim _{\epsilon \downarrow 0} \pi^{-1} \int_{\mu+\delta}^{\lambda+\delta} d \nu \operatorname{Im}\left[M_{p, q}\left(\nu+i \epsilon, x_{0}\right)\right], \\
& \lambda, \mu \in \mathbb{R}, 1 \leq p, q \leq 2,
\end{aligned}
$$

explicitly reads (cf., e.g., [32], Ch. 8)

$$
\begin{aligned}
& \frac{d \rho_{1,1}\left(\lambda, x_{0}\right)}{d \lambda}=\left\{\begin{array}{ll}
\frac{H_{g+1}\left(\lambda, x_{0}\right)}{2 \pi R_{2 g+1}(\lambda)^{1 / 2},} & \lambda \in \sigma(H)^{o} \\
0, & \lambda \in \mathbb{R} \backslash \sigma(H)
\end{array},\right. \\
& \frac{d \rho_{1,2}\left(\lambda, x_{0}\right)}{d \lambda}=\frac{d \rho_{2,1}\left(\lambda, x_{0}\right)}{d \lambda}=\left\{\begin{array}{ll}
\frac{-G_{g-1}\left(\lambda, x_{0}\right)}{2 \pi R_{2 g+1}(\lambda)^{1 / 2}}, & \lambda \in \sigma(H)^{o} \\
0, & \lambda \in \mathbb{R} \backslash \sigma(H)
\end{array},\right. \\
& \frac{d \rho_{2,2}\left(\lambda, x_{0}\right)}{d \lambda}= \begin{cases}\frac{F_{g}\left(\lambda, x_{0}\right)}{2 \pi R_{2 g+1}(\lambda)^{1 / 2}}, & \lambda \in \sigma(H)^{o} \\
0, & \lambda \in \mathbb{R} \backslash \sigma(H)\end{cases}
\end{aligned}
$$


(Here $A^{o}$ denotes the interior of $A \subset \mathbb{R}$.)

Closely associated with $H$ is $H_{x_{0}}^{\beta}$ in $L^{2}(\mathbb{R})$ defined by

$$
\begin{aligned}
H_{x_{0}}^{\beta} f= & \tau f, \beta \in \mathbb{R} \cup\{\infty\}, \quad x_{0} \in \mathbb{R}, \\
f \in \mathcal{D}\left(H_{x_{0}}^{\beta}\right)= & \left\{g \in L^{2}(\mathbb{R}) \mid g, g^{\prime} \in A C\left(\left[x_{0}, \pm R\right]\right) \text { for all } R>0,\right. \\
& \left.\lim _{\epsilon \downarrow 0}\left[g^{\prime}\left(x_{0} \pm \epsilon\right)+\beta g\left(x_{0} \pm \epsilon\right)\right]=0, \tau g \in L^{2}(\mathbb{R})\right\},
\end{aligned}
$$

with $A C_{\text {(loc) }}(I)$ the set of (locally) absolutely continuous functions on $I$. Here, in obvious notation, $\beta=\infty$ denotes the Dirichlet Schrödinger operator $H_{x_{0}}^{D}=H_{x_{0}}^{\infty}$ and $\beta=0$ the corresponding Neumann Schrödinger operator $H_{x_{0}}^{N}=H_{x_{0}}^{0}$. Moreover, $H_{x_{0}}^{\beta}$ decomposes into the direct sum of half-line operators

$$
H_{x_{0}}^{\beta}=H_{-, x_{0}}^{\beta} \oplus H_{+, x_{0}}^{\beta}, L^{2}(\mathbb{R})=L^{2}\left(\left(-\infty, x_{0}\right]\right) \oplus L^{2}\left(\left[x_{0}, \infty\right)\right) .
$$

The resolvent of $H_{x_{0}}^{\beta}$ reads

$$
\begin{gathered}
\left(\left(H_{x_{0}}^{\beta}-z\right)^{-1} f\right)(x)=\int_{\mathbb{R}} d x^{\prime} G_{x_{0}}^{\beta}\left(z, x, x^{\prime}\right) f\left(x^{\prime}\right), \quad z \in \mathbb{C} \backslash \sigma\left(H_{x_{0}}^{\beta}\right), f \in L^{2}(\mathbb{R}), \\
G_{x_{0}}^{\beta}\left(z, x, x^{\prime}\right)=G\left(z, x, x^{\prime}\right)-\frac{\left(\beta+\partial_{2}\right) G\left(z, x, x_{0}\right)\left(\beta+\partial_{1}\right) G\left(z, x_{0}, x^{\prime}\right)}{\left(\beta+\partial_{1}\right)\left(\beta+\partial_{2}\right) G\left(z, x_{0}, x_{0}\right)} \\
\beta \in \mathbb{R}, z \in \mathbb{C} \backslash\left\{\sigma\left(H_{x_{0}}^{\beta}\right) \cup \sigma(H)\right\}, \\
G_{x_{0}}^{\infty}\left(z, x, x^{\prime}\right)=G\left(z, x, x^{\prime}\right)-G\left(z, x, x_{0}\right) G\left(z, x_{0}, x^{\prime}\right) G\left(z, x_{0}, x_{0}\right)^{-1}, \\
z \in \mathbb{C} \backslash\left\{\sigma\left(H_{x_{0}}^{\infty}\right) \cup \sigma(H)\right\} .
\end{gathered}
$$

Next we define the polynomial $K_{g+1}^{\beta}(z, x), \beta \in \mathbb{R}$ of degree $g+1$ in $z$,

$$
K_{g+1}^{\beta}(z, x)=H_{g+1}(z, x)-2 \beta G_{g-1}(z, x)+\beta^{2} F_{g}(z, x)=\prod_{\ell=0}^{g}\left[z-\lambda_{\ell}^{\beta}(x)\right], \quad \beta \in \mathbb{R} .
$$

In particular,

$$
H_{g+1}(z, x)=K_{g+1}^{0}(z, x), \nu_{\ell}(x)=\lambda_{\ell}^{0}(x), \quad 0 \leq \ell \leq g .
$$

Explicitly, one computes

$$
\begin{aligned}
K_{1}^{\beta}= & \beta^{2}-V+z=\tilde{K}_{1}^{\beta}, \\
K_{2}^{\beta}= & c_{1}\left(\beta^{2}-V\right)+\frac{1}{4} V_{x x}-\frac{1}{2} V^{2}+\frac{1}{2} \beta V_{x}+\frac{1}{2} \beta^{2} V \\
& +\left(c_{1}+\beta^{2}-\frac{1}{2} V\right) z+z^{2}=c_{1} \tilde{K}_{1}^{\beta}+\tilde{K}_{2}^{\beta}, \\
K_{3}^{\beta}= & c_{2}\left(\beta^{2}-V\right)+c_{1}\left(\frac{1}{2} \beta^{2} V+\frac{1}{2} \beta V_{x}+\frac{1}{4} V_{x x}-\frac{1}{2} V^{2}\right)-\frac{1}{8} \beta V_{x x x} \\
& +\frac{3}{4} \beta V V_{x}-\frac{1}{8} \beta^{2} V_{x x}+\frac{3}{8} \beta^{2} V^{2}-\frac{1}{16} V_{x x x x}+\frac{3}{8} V_{x}^{2}+\frac{1}{2} V V_{x x}-\frac{3}{8} V^{3} \\
& +\left[\frac{1}{2} \beta V_{x}+\beta^{2} c_{1}+\frac{1}{2} \beta^{2} V-\frac{1}{2} c_{1} V+c_{2}+\frac{1}{8} V_{x x}-\frac{1}{8} V^{2}\right] z+\left[c_{1}+\beta^{2}-\frac{1}{2} V\right] z^{2}+z^{3} \\
= & c_{2} \tilde{K}_{1}^{\beta}+c_{1} \tilde{K}_{2}^{\beta}+\tilde{K}_{3}^{\beta},
\end{aligned}
$$

etc.

Then 3.35 and

$$
\left(\beta+\partial_{1}\right)\left(\beta+\partial_{2}\right) G(z, x, x)=\frac{i K_{g+1}^{\beta}(z, x)}{2 R_{2 g+1}(z)^{1 / 2}}, \quad \beta \in \mathbb{R}
$$


together with 3.47$)$ and $(3.48)$ yield

$$
\begin{aligned}
& \sigma\left(H_{x_{0}}^{\beta}\right)=\sigma(H) \cup\left\{\lambda_{\ell}^{\beta}\left(x_{0}\right)\right\}_{0 \leq \ell \leq g}, \quad \beta \in \mathbb{R}, \\
& \sigma\left(H_{x_{0}}^{\infty}\right)=\sigma(H) \cup\left\{\mu_{j}\left(x_{0}\right)\right\}_{1 \leq j \leq g}, \quad \mu_{j}\left(x_{0}\right)=\lambda_{j}^{\infty}\left(x_{0}\right), 1 \leq j \leq g,
\end{aligned}
$$

with

$$
\lambda_{0}^{\beta}\left(x_{0}\right) \leq E_{0}, \beta \in \mathbb{R}, \lambda_{\ell}^{\beta}\left(x_{0}\right) \in\left[E_{2 \ell-1}, E_{2 \ell}\right], 1 \leq \ell \leq g, \quad \beta \in \mathbb{R} \cup\{\infty\} .
$$

Next, one verifies

$$
\begin{gathered}
\phi(P, x)+\beta=\frac{i R_{2 g+1}^{1 / 2}(P)-G_{g-1}(\tilde{\pi}(P), x)+\beta F_{g}(\tilde{\pi}(P), x)}{F_{g}(\tilde{\pi}(P), x)} \\
=\frac{-K_{g+1}^{\beta}(\tilde{\pi}(P), x)}{i R_{2 g+1}^{1 / 2}(P)+G_{g-1}(\tilde{\pi}(P), x)-\beta F_{g}(\tilde{\pi}(P), x)}, \\
R_{2 g+1}(z)+\left[G_{g-1}(z, x)-\beta F_{g}(z, x)\right]^{2}=F_{g}(z, x) K_{g+1}^{\beta}(z, x), \\
{[\phi(P, x)+\beta]\left[\phi\left(P^{*}, x\right)+\beta\right]=K_{g+1}^{\beta}(z, x) / F_{g}(z, x),} \\
{\left[\psi_{x}\left(P, x, x_{0}\right)+\beta \psi\left(P, x, x_{0}\right)\right]\left[\psi_{x}\left(P^{*}, x, x_{0}\right)+\beta \psi\left(P^{*}, x, x_{0}\right)\right]=K_{g+1}^{\beta}(z, x) / F_{g}\left(z, x_{0}\right) .}
\end{gathered}
$$

The divisor of $\phi(., x)+\beta, \beta \in \mathbb{R}$ is given by

$$
(\phi(., x)+\beta)=\mathcal{D}_{\hat{\lambda}_{0}^{\beta}(x) \underline{\hat{\lambda}}^{\beta}(x)}-\mathcal{D}_{P_{\infty} \underline{\hat{\mu}}(x)},
$$

with

$$
\begin{aligned}
& R_{2 g+1}^{1 / 2}\left(\hat{\lambda}_{\ell}^{\beta}(x)\right)=-i G_{g-1}\left(\lambda_{\ell}^{\beta}(x), x\right)+i \beta F_{g}\left(\lambda_{\ell}^{\beta}(x), x\right), \\
& \hat{\lambda}_{\ell}^{\beta}(x)=\left(\lambda_{\ell}^{\beta}(x),-i G_{g-1}\left(\lambda_{\ell}^{\beta}(x), x\right)+i \beta F_{g}\left(\lambda_{\ell}^{\beta}(x), x\right)\right), 0 \leq \ell \leq g, \beta \in \mathbb{R} .
\end{aligned}
$$

The first-order system of differential equations for $\lambda_{\ell}^{\beta}(x), \beta \in \mathbb{R}$, i.e., the analog of (3.5) in the case $\beta=\infty$, will be derived in the next section (see 4.45 for $r=0$ ). Here we only record the final result for completeness,

$$
\begin{array}{r}
\lambda_{\ell}^{\beta \prime}(x)=-2 i\left[\beta^{2}-V(x)+\lambda_{\ell}^{\beta}(x)\right] R_{2 g+1}^{1 / 2}\left(\hat{\lambda}_{\ell}^{\beta}(x)\right) \prod_{\substack{m=0 \\
m \neq \ell}}^{g}\left[\lambda_{\ell}^{\beta}(x)-\lambda_{m}^{\beta}(x)\right]^{-1}, \\
\tilde{\pi}\left(\hat{\lambda}_{0}^{\beta}(x)\right)=\lambda_{0}^{\beta}(x) \leq E_{0}, \tilde{\pi}\left(\hat{\lambda}_{\ell}^{\beta}(x)\right)=\lambda_{\ell}^{\beta}(x) \in\left[E_{2 \ell-1}, E_{2 \ell}\right], 1 \leq \ell \leq g, \quad(\beta, x) \in \mathbb{R}^{2} .
\end{array}
$$

In particular, taking $\beta=0$ in (3.63) then yields the first-order system of differential equations for $\nu_{\ell}(x), 0 \leq \ell \leq g$. (We remark that $V(x)$ in (3.63) has been used for reasons of brevity only. In order to obtain a system of differential equations for $\lambda_{\ell}^{\beta}(x)$ one needs to replace $V(x)$ by the corresponding trace formula (see, e.g., (5.23), (5.30), and (5.34)).)

We emphasize that due to our convention $(\mathrm{A} .3)$ for $R_{2 g+1}^{1 / 2}(P)$, the differential equations (3.5) and 3.63 exhibit the well-known monotonicity properties of $\mu_{j}(x)$ and $\lambda_{j}^{\beta}(x), \beta \in \mathbb{R}$, $j \geq 1$ with respect to $x \in \mathbb{R}$. For instance, Dirichlet eigenvalues corresponding to the right (left) half axis $(x, \infty)((-\infty, x))$ associated with the decomposition (3.46) are always increasing (decreasing) with respect to $x \in \mathbb{R}$, etc. 
We conclude with the $\theta$-function representation for $\phi(P, x), \psi\left(P, x, x_{0}\right), V(x)$ to be derived in Section 4 (cf. Theorem 4.6) in the general $t$-dependent case.

$$
\begin{aligned}
& \phi(P, x)=-\beta+\frac{\theta\left(\underline{\Xi}_{P_{0}}-\underline{A}_{P_{0}}\left(P_{\infty}\right)+\underline{\alpha}_{P_{0}}(\underline{\hat{\mu}}(x))\right)}{\theta\left(\underline{\Xi}_{P_{0}}-\underline{A}_{P_{0}}\left(P_{\infty}\right)+\underline{\alpha}_{P_{0}}\left(\underline{\hat{\lambda}}^{\beta}(x)\right)\right)} \bullet \\
& \text { - } \frac{\theta\left(\Xi_{P_{0}}-\underline{A}_{P_{0}}(P)+\underline{\alpha}_{P_{0}}\left(\underline{\hat{\lambda}}^{\beta}(x)\right)\right)}{\theta\left(\underline{\Xi}_{P_{0}}-\underline{A}_{P_{0}}(P)+\underline{\alpha}_{P_{0}}(\underline{\hat{\mu}}(x))\right)} \exp \left[-\int_{P_{0}}^{P} \omega_{P_{\infty}, \hat{\lambda}_{0}^{\beta}(x)}^{(3)}\right] \text {, } \\
& \psi\left(P, x, x_{0}\right)=\frac{\theta\left(\underline{\Xi}_{P_{0}}-\underline{A}_{P_{0}}(P)+\underline{\alpha}_{P_{0}}(\underline{\hat{\mu}}(x))\right)}{\theta\left(\underline{\Xi}_{P_{0}}-\underline{A}_{P_{0}}\left(P_{\infty}\right)+\underline{\alpha}_{P_{0}}(\underline{\hat{\mu}}(x))\right)} \frac{\theta\left(\underline{\Xi}_{P_{0}}-\underline{A}_{P_{0}}\left(P_{\infty}\right)+\underline{\alpha}_{P_{0}}\left(\underline{\hat{\mu}}\left(x_{0}\right)\right)\right)}{\theta\left(\underline{\Xi}_{P_{0}}-\underline{A}_{P_{0}}(P)+\underline{\alpha}_{P_{0}}\left(\underline{\hat{\mu}}\left(x_{0}\right)\right)\right)} \bullet \\
& \text { - } \exp \left[-i\left(x-x_{0}\right) \int_{P_{0}}^{P} \omega_{P_{\infty}, 0}^{(2)}\right], \quad P_{0}=\left(E_{0}, 0\right), \beta \in \mathbb{R}
\end{aligned}
$$

with the linearizing property of the Abel map,

$$
\begin{aligned}
& \underline{\alpha}_{P_{0}}(\underline{\hat{\mu}}(x))=\underline{\alpha}_{P_{0}}\left(\underline{\hat{\mu}}\left(x_{0}\right)\right)+\frac{\underline{U}_{0}^{(2)}}{2 \pi}\left(x-x_{0}\right), \quad\left(x, x_{0}\right) \in \mathbb{R}^{2}, \\
& \underline{U}_{0}^{(2)}=\left(U_{0,1}^{(2)}, \ldots, U_{0, g}^{(2)}\right), U_{0, j}^{(2)}=\int_{b_{j}} \omega_{P_{\infty}, 0}^{(2)}, 1 \leq j \leq g .
\end{aligned}
$$

The Its-Matveev formula [26], [4], Ch. 3, [38], Ch. II for $V(x)$ then reads

$$
\begin{aligned}
& V(x)=E_{0}+\sum_{j=1}^{g}\left(E_{2 j-1}+E_{2 j}-2 \lambda_{j}\right)-2 \partial_{x}^{2} \ln \left[\theta\left(\underline{\Xi}_{P_{0}}-\underline{A}_{P_{0}}\left(P_{\infty}\right)+\underline{\alpha}_{P_{0}}(\underline{\hat{\mu}}(x))\right)\right] \\
& =E_{0}+\sum_{j=1}^{g}\left(E_{2 j-1}+E_{2 j}-2 \lambda_{j}\right)-2 \partial_{x}^{2} \ln \left[\theta\left(\underline{\Xi}_{P_{0}}+\underline{A}_{P_{0}}\left(\hat{\lambda}_{0}^{\beta}(x)\right)+\underline{\alpha}_{P_{0}}\left(\underline{\hat{\lambda}}^{\beta}(x)\right)\right)\right],
\end{aligned}
$$

where $\lambda_{j} \in\left[E_{2 j-1}, E_{2 j}\right], 1 \leq j \leq g$ are determined from

$$
\omega_{P_{\infty}, 0}^{(2)}=-\left[2 R_{2 g+1}^{1 / 2}(.)\right]^{-1} \prod_{j=1}^{g}\left(\tilde{\pi}-\lambda_{j}\right) d \tilde{\pi} \underset{\zeta \rightarrow 0}{=}\left[\zeta^{-2}+0(1)\right] d \zeta \text { near } P_{\infty}
$$

and the second equality in 3.68 is a consequence of the equivalence $\mathcal{D}_{P_{\infty} \underline{\hat{\mu}}(x)} \sim \mathcal{D}_{\hat{\lambda}_{0}^{\beta}(x) \hat{\hat{\lambda}}^{\beta}(x)}$, i.e.,

$$
\underline{A}_{P_{0}}\left(P_{\infty}\right)+\underline{\alpha}_{P_{0}}(\underline{\hat{\mu}}(x))=\underline{A}_{P_{0}}\left(\hat{\lambda}_{0}^{\beta}(x)\right)+\underline{\alpha}_{P_{0}}\left(\hat{\hat{\lambda}}^{\beta}(x)\right), x \in \mathbb{R} .
$$

\section{The Time-Dependent Formalism}

In this section we construct algebro-geometric solutions of the KdV hierarchy corresponding to $g$-gap initial values on the basis of a suitable time-dependent generalization of the polynomial approach developed in Chapters 2 and 3. Even though the final results (4.51)(4.55) are well-known, in fact, classical by now, the approach presented in this section, based on the fundamental meromorphic function $\phi(P, x, t)$ in $(4.15)$, merits attention since it easily extends to general $1+1$-dimensional completely integrable systems such as the AKNS and Toda hierarchies. (The corresponding approach to the Toda and Kac-van Moerbeke lattices 
is presented in detail in [5].) The results $4.36-(4.50)$ in connection with the general $\beta$ boundary condition in (3.45) and our strategy of proof of the theta function representation of the BA function $\psi\left(P, x, x_{0}, t, t_{0}\right)$ in (4.51), based on (4.15) and (4.16), are new.

Our starting point will be a $g$-gap solution $V^{(0)}$ of the stationary $\mathrm{KdV}_{g}$ equation,

$$
V^{(0)}(x)=E_{0}+\sum_{j=1}^{g}\left[E_{2 j-1}+E_{2 j}-2 \mu_{j}^{(0)}(x)\right], \mu_{j}^{(0)}(x) \in\left[E_{2 j-1}, E_{2 j}\right], \quad 1 \leq j \leq g
$$

satisfying

$$
\sum_{\ell=0}^{g} c_{g-\ell} f_{\ell+1, x}=0, \quad c_{0}=1,
$$

where $f_{\ell+1}$ are given by $(2.4)$ with $V=V^{(0)}$. Our principal aim then is to construct the $\mathrm{KdV}$ flow

$$
\mathrm{KdV}_{r}(V)=0, V\left(x, t_{0}\right)=V^{(0)}(x), \quad x \in \mathbb{R}
$$

for some $r \in \mathbb{N}_{0}$. In terms of Lax operators this amounts to solving

$$
\frac{d}{d t} L(t)-\left[P_{2 r+1}(t), L(t)\right]=0, t \in \mathbb{R},\left[P_{2 g+1}\left(t_{0}\right), L\left(t_{0}\right)\right]=0
$$

As a consequence one then obtains that

$$
\begin{gathered}
{\left[P_{2 g+1}(t), L(t)\right]=0, \quad t \in \mathbb{R},} \\
-P_{2 g+1}(t)^{2}=R_{2 g+1}(L(t))=\prod_{n=0}^{2 g}\left(L(t)-E_{n}\right), \quad t \in \mathbb{R}
\end{gathered}
$$

since the $\mathrm{KdV}_{r}$ flows are isospectral deformations of $L\left(t_{0}\right)$. In this paper we shall base the explicit solution of (4.3) not directly on (4.4) and (4.5) but instead take the following equations as our point of departure,

$$
\begin{aligned}
& V_{t}=-\frac{1}{2} \hat{F}_{r, x x x}+2(V-z) \hat{F}_{r, x}+V_{x} \hat{F}_{r}, \quad(x, t) \in \mathbb{R}^{2}, \\
& F_{g, x x} F_{g}-\frac{1}{2} F_{g, x}^{2}-2(V-z) F_{g}^{2}=2 R_{2 g+1}(z), \quad(x, t) \in \mathbb{R}^{2},
\end{aligned}
$$

where

$$
F_{g}(z, x, t)=\prod_{j=1}^{g}\left[z-\mu_{j}(x, t)\right]
$$

(cf. (2.14), 2.18), and (2.23)). In order to stress the fact that the integration constants $c_{\ell}$ used in $F_{g}$ and $F_{r}$ (cf. (2.10), 2.14) in general can differ from each other, we explicitly employ the notation $F_{g}, G_{g-1}, H_{g+1}, K_{g+1}^{\beta}$, etc. and $\hat{F}_{r}, \hat{G}_{r-1}, \hat{H}_{r+1}, \hat{K}_{r+1}^{\beta}$, etc. throughout this section. Similarly to 3.5 -3.8 , 3.11), and 3.12 we have

$$
\begin{gathered}
\mu_{j, x}(x, t)=-2 i R_{2 g+1}^{1 / 2}\left(\hat{\mu}_{j}(x, t)\right) \prod_{\substack{k=1 \\
k \neq j}}^{g}\left[\mu_{j}(x, t)-\mu_{k}(x, t)\right]^{-1}, 1 \leq j \leq g,(x, t) \in \mathbb{R}^{2}, \\
\left\{\hat{\mu}_{j}\left(x_{0}, t\right)\right\}_{1 \leq j \leq g} \subset K_{g}, \tilde{\pi}\left(\hat{\mu}_{j}\left(x_{0}, t\right)\right)=\mu_{j}\left(x_{0}, t\right) \in\left[E_{2 j-1}, E_{2 j}\right], 1 \leq j \leq g, t \in \mathbb{R}, \\
G_{g-1}(z, x, t)=-\frac{1}{2} F_{g, x}(z, x, t)=-i \sum_{j=1}^{g} R_{2 g+1}^{1 / 2}\left(\hat{\mu}_{j}(x, t)\right) \prod_{\substack{k=1 \\
k \neq j}}^{g}\left(\frac{z-\mu_{k}(x, t)}{\mu_{j}(x, t)-\mu_{k}(x, t)}\right),
\end{gathered}
$$




$$
\begin{aligned}
& R_{2 g+1}(z)+G_{g-1}(z, x, t)^{2}=F_{g}(z, x, t) H_{g+1}(z, x, t), \\
& H_{g+1}(z, x, t)=\prod_{\ell=0}^{g}\left[z-\nu_{\ell}(x, t)\right] .
\end{aligned}
$$

In analogy to (3.15) and (3.18) one then considers the meromorphic function $\phi(P, x, t)$ on $K_{g}$,

$$
\phi(P, x, t)=\frac{i R_{2 g+1}^{1 / 2}(P)-G_{g-1}(\tilde{\pi}(P), x, t)}{F_{g}(\tilde{\pi}(P), x, t)}=\frac{-H_{g+1}}{i R_{2 g+1}^{1 / 2}(P)+G_{g-1}(\tilde{\pi}(P), x, t)}, \quad(x, t) \in \mathbb{R}^{2}
$$

and the $t$-dependent BA function $\psi\left(P, x, x_{0}, t, t_{0}\right)$, meromorphic on $K_{g} \backslash\left\{P_{\infty}\right\}$,

$$
\begin{array}{r}
\psi\left(P, x, x_{0}, t, t_{0}\right)=\exp \left\{\int_{t_{0}}^{t} d s\left[\hat{F}_{r}\left(z, x_{0}, s\right) \phi\left(P, x_{0}, s\right)+\hat{G}_{r-1}\left(z, x_{0}, s\right)\right]+\int_{x_{0}}^{x} d y \phi(P, y, t)\right\} \\
\left(x, x_{0}, t, t_{0}\right) \in \mathbb{R}^{4} .
\end{array}
$$

Lemma 4.1. Let $P=\left(z, \sigma R_{2 g+1}(z)^{1 / 2}\right)=\left(\tilde{\pi}(P), R_{2 g+1}^{1 / 2}(P)\right) \in K_{g} \backslash\left\{P_{\infty}\right\},\left(z, x, x_{0}, t, t_{0}\right) \in$ $\mathbb{C} \times \mathbb{R}^{4}, r \in \mathbb{N}_{0}$. Then

(i). $\quad V(x, t)=E_{0}+\sum_{j=1}^{g}\left[E_{2 j-1}+E_{2 j}-2 \mu_{j}(x, t)\right]$.

(ii). $\phi(P, x, t)$ satisfies

$$
\begin{aligned}
& \phi_{x}(P, x, t)+\phi(P, x, t)^{2}=V(x, t)-z \\
& \phi_{t}(P, x, t)=\partial_{x}\left[\hat{F}_{r}(z, x, t) \phi(P, x, t)+\hat{G}_{r-1}(z, x, t)\right] .
\end{aligned}
$$

(iii). $\psi\left(P, x, x_{0}, t, t_{0}\right)$ satisfies

$$
\begin{aligned}
& -\psi_{x x}\left(P, x, x_{0}, t, t_{0}\right)+[V(x, t)-z] \psi\left(P, x, x_{0}, t, t_{0}\right)=0, \\
& \psi_{t}\left(P, x, x_{0}, t, t_{0}\right)=\hat{F}_{r}(z, x, t) \psi_{x}\left(P, x, x_{0}, t, t_{0}\right)+\hat{G}_{r-1}(z, x, t) \psi\left(P, x, x_{0}, t, t_{0}\right) .
\end{aligned}
$$

(iv). $\phi(P, x, t) \phi\left(P^{*}, x, t\right)=H_{g+1}(z, x, t) / F_{g}(z, x, t)$.

(v). $\phi(P, x, t)+\phi\left(P^{*}, x, t\right)=-2 G_{g-1}(z, x, t) / F_{g}(z, x, t)=F_{g, x}(z, x, t) / F_{g}(z, x, t)$.

(vi). $\phi(P, x, t)-\phi\left(P^{*}, x, t\right)=2 i R_{2 g+1}^{1 / 2}(P) / F_{g}(z, x, t)$.

Proof. The proof of (i), 4.18), 4.20), and (iv)-(vi) is analogous to that in Lemma 3.1. In order to prove 4.19) one can argue as follows. By 4.7) and 4.18,

$$
\partial_{t}\left(\phi_{x}+\phi^{2}\right)=\phi_{t x}+2 \phi \phi_{t}=V_{t}=\left(\hat{F}_{r} \phi+\hat{G}_{r-1}\right)_{x x}+2 \phi\left(\hat{F}_{r} \phi+\hat{G}_{r-1}\right)_{x},
$$

which implies

$$
\left(\partial_{x}+2 \phi\right)\left[\phi_{t}-\left(\hat{F}_{r} \phi+\hat{G}_{r-1}\right)_{x}\right]=0 \text { and hence } \phi_{t}=\left(\hat{F}_{r} \phi+\hat{G}_{r-1}\right)_{x}+C e^{-2 \int^{x} d y \phi},
$$

where $C$ is independent of $x$ (but may depend on $P$ and $t$ ). The high-energy behavior of $\phi(P, x, t)$ derived from 4.15 yields $\phi(P, x, t) \underset{z \rightarrow \infty}{=} \pm i(z)^{1 / 2}+0(1), P \in \Pi_{ \pm}$uniformly in $(x, t) \in \mathbb{R}^{2}$ and hence $C=0$ proving 4.19. 4.21 then immediately follows from (4.16) and (4.19). 
In analogy to 3.28 we now introduce

$$
\hat{H}_{r+1}(z, x, t)=\frac{1}{2} \hat{F}_{r, x x}(z, x, t)-[V(x, t)-z] \hat{F}_{r}(z, x, t) .
$$

From 4.7) and 4.8 one then computes

$$
\hat{H}_{r+1, x}=\frac{1}{2} \hat{F}_{r, x x x}-(V-z) \hat{F}_{r, x}-V_{x} \hat{F}_{r}=-V_{t}-2(V-z) \hat{G}_{r-1} .
$$

The $t$-dependence of $F_{g}, G_{g-1}$, and $H_{g+1}$ is governed by

Lemma 4.2. Let $(z, x, t) \in \mathbb{C} \times \mathbb{R}^{2}, r \in \mathbb{N}_{0}$. Then

$$
\begin{array}{ll}
\text { (i). } & F_{g, t}(z, x, t)=2\left[F_{g}(z, x, t) \hat{G}_{r-1}(z, x, t)-\hat{F}_{r}(z, x, t) G_{g-1}(z, x, t)\right] . \\
\text { (ii). } & G_{g-1, t}(z, x, t)=F_{g}(z, x, t) \hat{H}_{r+1}(z, x, t)-\hat{F}_{r}(z, x, t) H_{g+1}(z, x, t) . \\
\text { (iii). } & H_{g+1, t}(z, x, t)=2\left[\hat{H}_{r+1}(z, x, t) G_{g-1}(z, x, t)-H_{g+1}(z, x, t) \hat{G}_{r-1}(z, x, t)\right] .
\end{array}
$$

Proof. By (2.17), 4.19), and (4.24),

$$
\begin{aligned}
& \phi_{t}(P)-\phi_{t}\left(P^{*}\right)=-2 i R_{2 g+1}^{1 / 2}(P) F_{g}^{-2} F_{g, t} \\
& =\partial_{x}\left[\hat{F}_{r}\left(\phi(P)-\phi\left(P^{*}\right)\right)\right]=2 i R_{2 g+1}^{1 / 2}(P)\left(F_{g} \hat{F}_{r, x}-F_{g, x} \hat{F}_{r}\right) F_{g}^{-2},
\end{aligned}
$$

implying 4.27). Similarly, by 2.17) and 4.25),

$$
G_{g-1, t}=-\frac{1}{2} F_{g, t x}=\hat{F}_{r} G_{g-1, x}-F_{g} \hat{G}_{r-1, x}=F_{g} \hat{H}_{r+1}-\hat{F}_{r} H_{g+1} .
$$

Finally, 2.17), 4.25)-4.28) yield

$$
\begin{aligned}
& H_{g+1, t}=-G_{g-1, t x}-(V-z) F_{g, t}-V_{t} F_{g}=-F_{g, x} \hat{H}_{r+1}-F_{g} \hat{H}_{r+1, x}+\hat{F}_{r, x} H_{g+1}+\hat{F}_{r} H_{g+1, x} \\
& \quad-2(V-z)\left(F_{g} \hat{G}_{r-1}-\hat{F}_{r} G_{g-1}\right)-V_{t} F_{g}=2\left(G_{g-1} \hat{H}_{r+1}-2 \hat{G}_{r-1} H_{g+1}\right) . \quad \square
\end{aligned}
$$

As a consequence of (4.27) one obtains the following time-dependence of $\mu_{j}(x, t)$.

Corollary 4.3. Let $(x, t) \in \mathbb{R}^{2}, r \in \mathbb{N}_{0}$. Then

$$
\begin{aligned}
\mu_{j, t}(x, t) & =-2 i \hat{F}_{r}\left(\mu_{j}(x, t), x\right) R_{2 g+1}^{1 / 2}\left(\hat{\mu}_{j}(x, t)\right) \prod_{\substack{k=1 \\
k \neq j}}^{g}\left[\mu_{j}(x, t)-\mu_{k}(x, t)\right]^{-1}, \\
\hat{\mu}_{j}\left(x, t_{0}\right) & =\hat{\mu}_{j}^{(0)}(x), \quad 1 \leq j \leq g, \\
\tilde{\pi}\left(\hat{\mu}_{j}(x, t)\right) & =\mu_{j}(x, t) \in\left[E_{2 j-1}, E_{2 j}\right], \quad 1 \leq j \leq g .
\end{aligned}
$$

Proof. Take $z=\mu_{j}(x, t)$ in (4.27) and observe (4.1), 4.3), (4.9), and

$$
R_{2 g+1}^{1 / 2}\left(\hat{\mu}_{j}(x, t)\right)=i G_{g-1}\left(\mu_{j}(x, t), x, t\right), \hat{\mu}_{j}(x, t)=\left(\mu_{j}(x, t), i G_{g-1}\left(\mu_{j}(x, t), x, t\right)\right),
$$

the latter fact following from (4.12) (as in (3.9)).

One observes that the special case $r=0$ (i.e., $\hat{F}_{0}=1$ ) in 4.30 is equivalent to 4.10 , 4.11.

Next we record the remaining $t$-dependent analogs of Lemma 3.1 (vii)-(ix). 
Lemma 4.4. Let $P=\left(z, \sigma R_{2 g+1}(z)^{1 / 2}\right)=\left(\tilde{\pi}(P), R_{2 g+1}^{1 / 2}(P)\right) \in K_{g} \backslash\left\{P_{\infty}\right\},\left(z, x, x_{0}, t, t_{0}\right) \in$ $\mathbb{C} \times \mathbb{R}^{4}, r \in \mathbb{N}_{0}$. Then

(i). $\quad \psi\left(P, x, x_{0}, t, t_{0}\right) \psi\left(P^{*}, x, x_{0}, t, t_{0}\right)=F_{g}(z, x, t) / F_{g}\left(z, x_{0}, t_{0}\right)$.

(ii). $\quad \psi_{x}\left(P, x, x_{0}, t, t_{0}\right) \psi_{x}\left(P^{*}, x, x_{0}, t, t_{0}\right)=H_{g+1}(z, x, t) / F_{g}\left(z, x_{0}, t_{0}\right)$.

(iii). $\quad \psi\left(P, x, x_{0}, t, t_{0}\right)=\left[F_{g}(z, x, t) / F_{g}\left(z, x_{0}, t_{0}\right)\right]^{1 / 2}$

$$
\text { - } \exp \left\{i R_{2 g+1}^{1 / 2}(P)\left[\int_{t_{0}}^{t} d s \hat{F}_{r}\left(z, x_{0}, s\right) F_{g}\left(z, x_{0}, s\right)^{-1}+\int_{x_{0}}^{x} d y F_{g}(z, y, t)^{-1}\right]\right\} \text {. }
$$

Proof. (i). Combining 4.16), 4.23), and 4.27) yields

$$
\begin{aligned}
& \psi\left(P, x, x_{0}, t, t_{0}\right) \psi\left(P^{*}, x, x_{0}, t, t_{0}\right) \\
= & \exp \left[\int_{t_{0}}^{t} d s F_{g, s}\left(z, x_{0}, s\right) F_{g}\left(z, x_{0}, s\right)^{-1}+\int_{x_{0}}^{x} d y F_{g, y}(z, y, t) F_{g}(z, y, t)^{-1}\right] \\
= & {\left[F_{g}\left(z, x_{0}, t\right) / F_{g}\left(z, x_{0}, t_{0}\right)\right]\left[F_{g}(z, x, t) / F_{g}\left(z, x_{0}, t\right)\right]=F_{g}(z, x, t) / F_{g}\left(z, x_{0}, t_{0}\right) . }
\end{aligned}
$$

(ii). 4.22, 4.33 and $\psi_{x}=\phi \psi$ imply

$$
\begin{aligned}
& \psi_{x}\left(P, x, x_{0}, t, t_{0}\right) \psi_{x}\left(P^{*}, x, x_{0}, t, t_{0}\right) \\
= & {\left[H_{g+1}(z, x, t) / F_{g}(z, x, t)\right]\left[F_{g}(z, x, t) / F_{g}\left(z, x_{0}, t_{0}\right)\right]=H_{g+1}(z, x, t) / F_{g}\left(z, x_{0}, t_{0}\right) . }
\end{aligned}
$$

(iii). Follows from 4.15), 4.16), and (4.27).

Remark 4.5. We emphasize that instead of taking (4.7) and (4.8) as our starting point for solving (4.3), and subsequently deriving the first-order differential system (4.10), (4.30), one could have started directly with the system (4.10), 4.30) and derived (4.7), (4.8) and the remaining facts of this section (cf. [5]).

Next, we turn to the $t$-dependent analog of $3.50-3.63$ and start by introducing

$$
K_{g+1}^{\beta}(z, x, t)=H_{g+1}(z, x, t)-2 \beta G_{g-1}(z, x, t)+\beta^{2} F_{g}(z, x, t)=\prod_{\ell=0}^{g}\left[z-\lambda_{\ell}^{\beta}(x, t)\right], \quad \beta \in \mathbb{R},
$$

with

$$
H_{g+1}(z, x, t)=K_{g+1}^{0}(z, x, t), \nu_{\ell}(x, t)=\lambda_{\ell}^{0}(x, t), \quad 0 \leq \ell \leq g .
$$

One then verifies in analogy to 3.57-3.62 that

$$
\begin{array}{r}
\phi(P, x, t)+\beta=\frac{i R_{2 g+1}^{1 / 2}(P)-G_{g-1}(\tilde{\pi}(P), x, t)+\beta F_{g}(\tilde{\pi}(P), x, t)}{F_{g}(\tilde{\pi}(P), x, t)} \\
=\frac{-K_{g+1}^{\beta}(\tilde{\pi}(P), x, t)}{i R_{2 g+1}^{1 / 2}(P)+G_{g-1}(\tilde{\pi}(P), x, t)-\beta F_{g}(\tilde{\pi}(P), x, t)}, \\
R_{2 g+1}(z)+\left[G_{g-1}(z, x, t)-\beta F_{g}(z, x, t)\right]^{2}=F_{g}(z, x, t) K_{g+1}^{\beta}(z, x, t), \\
{[\phi(P, x, t)+\beta]\left[\phi\left(P^{*}, x, t\right)+\beta\right]=K_{g+1}^{\beta}(z, x, t) / F_{g}(z, x, t),}
\end{array}
$$




$$
\begin{aligned}
& {\left[\psi_{x}\left(P, x, x_{0}, t, t_{0}\right)+\beta \psi\left(P, x, x_{0}, t, t_{0}\right)\right]\left[\psi_{x}\left(P^{*}, x, x_{0}, t, t_{0}\right)+\beta \psi\left(P^{*}, x, x_{0}, t, t_{0}\right)\right]} \\
& =K_{g+1}^{\beta}(z, x, t) / F_{g}\left(z, x_{0}, t_{0}\right), \\
& (\phi(., x, t)+\beta)=\mathcal{D}_{\hat{\lambda}_{0}^{\beta}(x, t) \underline{\hat{\lambda}}^{\beta}(x, t)}-\mathcal{D}_{P_{\infty} \underline{\mu}(x, t)},
\end{aligned}
$$

with

$$
\begin{aligned}
& R_{2 g+1}^{1 / 2}\left(\hat{\lambda}_{\ell}^{\beta}(x, t)\right)=-i G_{g-1}\left(\lambda_{\ell}^{\beta}(x, t), x, t\right)+i \beta F_{g}\left(\lambda_{\ell}^{\beta}(x, t), x, t\right), \\
& \hat{\lambda}_{\ell}^{\beta}(x, t)=\left(\lambda_{\ell}^{\beta}(x, t),-i G_{g-1}\left(\lambda_{\ell}^{\beta}(x, t), x, t\right)+i \beta F_{g}\left(\lambda_{\ell}^{\beta}(x, t), x, t\right)\right), \quad 0 \leq \ell \leq g .
\end{aligned}
$$

Eq. (4.36) and Lemma 4.2 then yield

$$
\begin{aligned}
K_{g+1, t}^{\beta}(z, x, t)= & 2\left\{\hat{K}_{r+1}^{\beta}(z, x, t)\left[G_{g-1}(z, x, t)-\beta F_{g}(z, x, t)\right]\right. \\
& \left.-K_{g+1}^{\beta}(z, x, t)\left[\hat{G}_{r-1}(z, x, t)-\beta \hat{F}_{r}(z, x, t)\right]\right\}
\end{aligned}
$$

and in analogy to Corollary 4.3 one obtains from (4.44),

$$
\begin{gathered}
\lambda_{\ell, t}^{\beta}(x, t)=-2 i \hat{K}_{r+1}^{\beta}\left(\lambda_{\ell}^{\beta}(x, t), x, t\right) R_{2 g+1}^{1 / 2}\left(\hat{\lambda}_{\ell}^{\beta}(x, t)\right) \prod_{\substack{m=0 \\
m \neq \ell}}^{g}\left[\lambda_{\ell}^{\beta}(x, t)-\lambda_{m}^{\beta}(x, t)\right]^{-1}, \\
\hat{\lambda}_{\ell}^{\beta}\left(x, t_{0}\right)=\hat{\lambda}_{\ell}^{\beta,(0)}(x), \quad 0 \leq \ell \leq g,(x, t) \in \mathbb{R}^{2}, \\
\tilde{\pi}\left(\hat{\lambda}_{0}^{\beta}(x, t)\right)=\lambda_{0}^{\beta}(x, t) \leq E_{0}, \tilde{\pi}\left(\hat{\lambda}_{\ell}^{\beta}(x, t)\right)=\lambda_{\ell}^{\beta}(x, t) \in\left[E_{2 \ell-1}, E_{2 \ell}\right], \quad(x, t) \in \mathbb{R}^{2},
\end{gathered}
$$

where $\left\{\lambda_{\ell}^{\beta,(0)}(y)\right\}_{0 \leq \ell \leq g}$ are the corresponding eigenvalues of $H_{y}^{\beta,(0)}$ (cf. 3.45), 3.54), and (3.56) associated with the initial value $V^{(0)}(x)$ in (4.1).

In an analogous fashion one can analyze the behavior of $\lambda_{\ell}^{\beta}(x, t)$ as a function of $\beta \in \mathbb{R}$. In fact, 4.36 yields

$$
\frac{\partial}{\partial \beta} K_{g+1}^{\beta}(z, x, t)=-2\left[G_{g-1}(z, x, t)-\beta F_{g}(z, x, t)\right]
$$

and hence

$$
\begin{aligned}
& \left.\frac{\partial}{\partial \beta} K_{g+1}^{\beta}(z, x, t)\right|_{z=\lambda_{\ell}^{\beta}(x, t)}=-\left[\frac{\partial}{\partial \beta} \lambda_{\ell}^{\beta}(x, t)\right] \prod_{\substack{m=0 \\
m \neq \ell}}^{g}\left[\lambda_{\ell}^{\beta}(x, t)-\lambda_{m}^{\beta}(x, t)\right] \\
& =-2\left[G_{g-1}\left(\lambda_{\ell}^{\beta}(x, t), x, t\right)-\beta F_{g}\left(\lambda_{\ell}^{\beta}(x, t), x, t\right)\right]=-2 i R_{2 g+1}^{1 / 2}\left(\hat{\lambda}_{\ell}^{\beta}(x, t)\right)
\end{aligned}
$$

by (4.43). This implies for $(\beta, x, t) \in \mathbb{R}^{3}$,

$$
\frac{\partial}{\partial \beta} \lambda_{\ell}^{\beta}(x, t)=2 i R_{2 g+1}^{1 / 2}\left(\hat{\lambda}_{\ell}^{\beta}(x, t)\right) \prod_{\substack{m=0 \\ m \neq \ell}}^{g}\left[\lambda_{\ell}^{\beta}(x, t)-\lambda_{m}^{\beta}(x, t)\right]^{-1}, \quad 0 \leq \ell \leq g .
$$

As in Section 3 we conclude with the $\theta$-function representation of $\phi(P, x, t), \psi\left(P, x, x_{0}, t, t_{0}\right)$, and $V(x, t)$. 
Theorem 4.6. Let $P=\left(z, \sigma R_{2 g+1}(z)^{1 / 2}\right) \in K_{g} \backslash\left\{P_{\infty}\right\},\left(z, x, x_{0}, t, t_{0}\right) \in \mathbb{C} \times \mathbb{R}^{4}, P_{0}=\left(E_{0}, 0\right)$. Then

$$
\begin{aligned}
\phi(P, x, t)=-\beta+\frac{\theta\left(\underline{\Xi}_{P_{0}}-\right.}{\left.-\underline{A}_{P_{0}}\left(P_{\infty}\right)+\underline{\alpha}_{P_{0}}(\underline{\hat{\mu}}(x, t))\right)} & \bullet \\
\theta\left(\underline{\Xi}_{P_{0}}-\right. & \left.\underline{A}_{P_{0}}\left(P_{\infty}\right)+\underline{\alpha}_{P_{0}}\left(\underline{\hat{\lambda}}^{\beta}(x, t)\right)\right) \\
& \bullet \frac{\theta\left(\underline{\Xi}_{P_{0}}-\underline{A}_{P_{0}}(P)+\underline{\alpha}_{P_{0}}\left(\underline{\hat{\lambda}}^{\beta}(x, t)\right)\right)}{\theta\left(\underline{\Xi}_{P_{0}}-\underline{A}_{P_{0}}(P)+\underline{\alpha}_{P_{0}}(\underline{\hat{\mu}}(x, t))\right)} \exp \left[-\int_{P_{0}}^{P} \omega_{P_{\infty}, \hat{\lambda}_{0}^{\beta}(x, t)}^{(3)}\right]
\end{aligned}
$$

and

$$
\begin{aligned}
& \psi\left(P, x, x_{0}, t, t_{0}\right)=\frac{\theta\left(\underline{\Xi}_{P_{0}}-\underline{A}_{P_{0}}(P)+\underline{\alpha}_{P_{0}}(\underline{\hat{\mu}}(x, t))\right)}{\theta\left(\underline{\Xi}_{P_{0}}-\underline{A}_{P_{0}}\left(P_{\infty}\right)+\underline{\alpha}_{P_{0}}(\underline{\hat{\mu}}(x, t))\right)} \bullet \\
& \text { - } \frac{\theta\left(\underline{\Xi}_{P_{0}}-\underline{A}_{P_{0}}\left(P_{\infty}\right)+\underline{\alpha}_{P_{0}}\left(\underline{\hat{\mu}}\left(x_{0}, t_{0}\right)\right)\right)}{\theta\left(\underline{\Xi}_{P_{0}}-\underline{A}_{P_{0}}(P)+\underline{\alpha}_{P_{0}}\left(\underline{\hat{\mu}}\left(x_{0}, t_{0}\right)\right)\right)} \exp \left[-i\left(x-x_{0}\right) \int_{P_{0}}^{P} \omega_{P_{\infty}, 0}^{(2)}-i\left(t-t_{0}\right) \int_{P_{0}}^{P} \Omega_{P_{\infty}, 2 r}^{(2)}\right] \text {, }
\end{aligned}
$$

where (cf. A.26)

$$
\begin{aligned}
& \Omega_{P_{\infty}, 2 r}^{(2)}=\sum_{s=0}^{r} c_{r-s}(2 s+1) \omega_{P_{\infty}, 2 s}^{(2)}, \\
& \underline{\alpha}_{P_{0}}(\underline{\hat{\mu}}(x, t))=\underline{\alpha}_{P_{0}}\left(\underline{\hat{\mu}}\left(x_{0}, t_{0}\right)\right)+\frac{\underline{U}_{0}^{(2)}}{2 \pi}\left(x-x_{0}\right)+\frac{\underline{U}_{2 r}^{(2)}}{2 \pi}\left(t-t_{0}\right), \\
& \underline{U}_{2 r}^{(2)}=\left(U_{2 r, 1}^{(2)}, \ldots, U_{2 r, g}^{(2)}\right), U_{2 r, j}^{(2)}=\int_{b_{j}} \Omega_{P_{\infty}, 2 r}^{(2)}, \quad 1 \leq j \leq g .
\end{aligned}
$$

The Its-Matveev formula (26], [4], Ch. 3, [38], Ch. II) for $V(x, t)$ reads (cf. (3.68))

$$
V(x, t)=E_{0}+\sum_{j=1}^{g}\left(E_{2 j-1}+E_{2 j}-2 \lambda_{j}\right)-2 \partial_{x}^{2} \ln \left[\theta\left(\Xi_{P_{0}}-\underline{A}_{P_{0}}\left(P_{\infty}\right)+\underline{\alpha}_{P_{0}}(\underline{\hat{\mu}}(x, t))\right)\right] .
$$

Sketch of Proof. Since 4.50) follows directly from 4.42 and $(\mathrm{A} .29$, and 4.55 can be inferred from (4.51) and (4.20) upon expanding all quantities in (4.20) near $P_{\infty}$ in a wellknown manner, we first concentrate on the proof of 4.51$)$. Let $\psi\left(P, x, x_{0}, t, t_{0}\right)$ be defined as in (4.16) and denote the right-hand-side of (4.51) by $\Psi\left(P, x, x_{0}, t, t_{0}\right)$. In order to prove that $\psi=\Psi$, one first observes from 4.10 and 4.30 that

$$
\hat{F}_{r}\left(\tilde{\pi}(P), x_{0}, s\right) \phi\left(P, x_{0}, s\right)=\frac{\partial}{\partial s} \ln \left[\mu_{j}\left(x_{0}, s\right)-\tilde{\pi}(P)\right]+0(1) \text { for } P \text { near } \hat{\mu}_{j}\left(x_{0}, s\right)
$$

and

$$
\phi(P, y, t)=\frac{\partial}{\partial y} \ln \left[\mu_{j}(y, t)-\tilde{\pi}(P)\right]+0(1) \text { for } P \text { near } \hat{\mu}_{j}(y, t)
$$

Hence

$$
\begin{aligned}
& \exp \left\{\int_{t_{0}}^{t} d s\left[\frac{\partial}{\partial s} \ln \left(\mu_{j}\left(x_{0}, s\right)-\tilde{\pi}(P)\right)+0(1)\right]\right\} \\
& = \begin{cases}{\left[\mu_{j}\left(x_{0}, t\right)-\tilde{\pi}(P)\right] 0(1)} & \text { for } P \text { near } \hat{\mu}_{j}\left(x_{0}, t\right) \neq \hat{\mu}_{j}\left(x_{0}, t_{0}\right) \\
0(1) & \text { for } P \text { near } \hat{\mu}_{j}\left(x_{0}, t\right)=\hat{\mu}_{j}\left(x_{0}, t_{0}\right) \\
{\left[\mu_{j}\left(x_{0}, t_{0}\right)-\tilde{\pi}(P)\right]^{-1} 0(1)} & \text { for } P \text { near } \hat{\mu}_{j}\left(x_{0}, t_{0}\right) \neq \hat{\mu}_{j}\left(x_{0}, t\right)\end{cases}
\end{aligned}
$$


and

$$
\begin{aligned}
& \exp \left\{\int_{x_{0}}^{x} d y\left[\frac{\partial}{\partial y} \ln \left(\mu_{j}(y, t)-\tilde{\pi}(P)\right)+0(1)\right]\right\} \\
& = \begin{cases}{\left[\mu_{j}(x, t)-\tilde{\pi}(P)\right] 0(1)} & \text { for } P \text { near } \hat{\mu}_{j}(x, t) \neq \hat{\mu}_{j}\left(x_{0}, t\right) \\
0(1) & \text { for } P \text { near } \hat{\mu}_{j}(x, t)=\hat{\mu}_{j}\left(x_{0}, t\right), \\
{\left[\mu_{j}\left(x_{0}, t\right)-\tilde{\pi}(P)\right]^{-1} 0(1)} & \text { for } P \text { near } \hat{\mu}_{j}\left(x_{0}, t\right) \neq \hat{\mu}_{j}(x, t)\end{cases}
\end{aligned}
$$

where $0(1) \neq 0$ in (4.58) and (4.59). Consequently, all zeros and poles of $\psi$ and $\Psi$ on $K_{g} \backslash\left\{P_{\infty}\right\}$ are simple and coincide. By an application of the Riemann-Roch theorem it remains to identify the essential singularity of $\psi$ and $\Psi$ at $P_{\infty}$. For that purpose we first recall the known fact that the diagonal Green's function $G(z, x, x, t)$ of $H(t)$ satisfies

$$
G(z, x, x, t) \underset{\zeta \rightarrow 0}{=}(i / 2) \zeta \sum_{j=0}^{\infty} \tilde{f}_{j}(x, t) \zeta^{2 j}, \quad \zeta=1 / \sqrt{z},
$$

with $\tilde{f}_{j}(x, t)$ the homogeneous coefficients as introduced in the context of $(2.12)$ satisfying the recursion (2.4) for all $j \in \mathbb{N}$. Combining

$$
G(z, x, x, t)=\frac{i F_{g}(z, x, t)}{2 R_{2 g+1}(z)^{1 / 2}}
$$

(cf. (3.35), 4.15 , 4.16), and (4.60) then yields

$$
\int_{x_{0}}^{x} d y \phi(P, y, t) \underset{\zeta \rightarrow 0}{=} \int_{x_{0}}^{x} d y \frac{i R_{2 g+1}^{1 / 2}(P)}{F_{g}(\tilde{\pi}(P), y, t)}+0\left(\zeta^{2}\right) \underset{\zeta \rightarrow 0}{=} i\left(x-x_{0}\right)\left[\zeta^{-1}+0(1)\right],
$$

which coincides with the singularity at $P_{\infty}$ of the $x$-dependent term in the exponent of (4.51) taking into account (3.69). Finally, in order to identify the $t$-dependent essential singularity of $\psi$ and $\Psi$, we may allude to (2.20) and, without loss of generality, consider the homogeneous case where $c_{0}=1, c_{q}=0,1 \leq q \leq r$. Invoking (4.27) then yields from 4.15) and (4.61)

$$
\begin{aligned}
& \int_{t_{0}}^{t} d s\left[\tilde{F}_{r}\left(z, x_{0}, s\right) \phi\left(P, x_{0}, s\right)+\tilde{G}_{r-1}\left(z, x_{0}, s\right)\right] \\
& =\int_{t_{0}}^{t} d s\left\{\tilde{F}_{r}\left(z, x_{0}, s\right) i R_{2 g+1}^{1 / 2}(P) F_{g}\left(z, x_{0}, s\right)^{-1}+\frac{1}{2} \frac{\partial}{\partial s} \ln \left[F_{g}\left(z, x_{0}, s\right)\right]\right\} \\
& \underset{\zeta \rightarrow 0}{=}-\frac{1}{2} \int_{t_{0}}^{t} d s \tilde{F}_{r}\left(z, x_{0}, s\right) G\left(z, x_{0}, x_{0}, s\right)^{-1}+0(1), \quad \zeta=1 / \sqrt{z} .
\end{aligned}
$$

Comparing (2.14) (in the homogeneous case) and 4.60 implies

$$
-\frac{1}{2} \tilde{F}_{r}\left(z, x_{0}, s\right) G\left(z, x_{0}, x_{0}, s\right)^{-1} \underset{\zeta \rightarrow 0}{=} i \zeta^{-2 r-1}+0(1)
$$

and hence

$$
\int_{t_{0}}^{t} d s\left[\tilde{F}_{r}\left(z, x_{0}, s\right) \phi\left(P, x_{0}, s\right)+\tilde{G}_{r-1}\left(z, x_{0}, s\right)\right] \underset{\zeta \rightarrow 0}{=} i\left(t-t_{0}\right)\left[\zeta^{-2 r-1}+0(1)\right],
$$

completing the proof of (4.51). The linearity of the Abel map with respect to $x$ and $t$ in 4.53) then follows by a standard argument considering the differential $\Omega\left(x, x_{0}, t, t_{0}\right)=$ $d \ln \psi\left(., x, x_{0}, t, t_{0}\right)$. 


\section{General Trace Formulas}

Following a recent series of papers on new trace formulas for Schrödinger operators [16][19], 22]-24], [39], we first discuss appropriate Krein spectral shift functions, the key tool for general higher-order trace formulas. Subsequently, we develop a new method for deriving small-time heat kernel (respectively high-energy resolvent) expansion coefficients associated with the general $\beta$-boundary conditions in (5.3). Interest in these types of trace formulas stems from their crucial role in the solution of inverse spectral problems.

Unlike Sections 3 and 4, where we focused on the special case of stationary finite-gap solutions of the KdV hierarchy (the natural extension of solitons as reflectionless potentials), we now turn to the general situation and consider potentials of the type

$$
V \in C^{\infty}(\mathbb{R}), V(x) \geq c, x \in \mathbb{R}, V \text { real-valued. }
$$

As in Section 3 we introduce the differential expression $\tau=-\frac{d^{2}}{d x^{2}}+V(x), x \in \mathbb{R}$ and define the self-adjoint operators $H$ and $H_{x_{0}}^{\beta}$ in $L^{2}(\mathbb{R})$,

$$
H f=\tau f, f \in \mathcal{D}(H)=\left\{g \in L^{2}(\mathbb{R}) \mid g, g^{\prime} \in A C_{\mathrm{loc}}(\mathbb{R}) ; \tau g \in L^{2}(\mathbb{R})\right\}
$$

and for $\beta \in \mathbb{R} \cup\{\infty\}, x_{0} \in \mathbb{R}$,

$$
\begin{array}{r}
H_{x_{0}}^{\beta} f=\tau f, f \in \mathcal{D}\left(H_{x_{0}}^{\beta}\right)=\left\{f \in L^{2}(\mathbb{R}) \mid g, g^{\prime} \in A C\left(\left[x_{0}, \pm R\right]\right) \text { for all } R>0,\right. \\
\left.\qquad \lim _{\epsilon \downarrow 0}\left[g^{\prime}\left(x_{0} \pm \epsilon\right)+\beta g\left(x_{0} \pm \epsilon\right)\right]=0, \quad \tau g \in L^{2}(\mathbb{R})\right\},
\end{array}
$$

with $H_{x_{0}}^{\infty}=H_{x_{0}}^{D}\left(H_{x_{0}}^{0}=H_{x_{0}}^{N}\right)$ the corresponding Dirichlet (Neumann) Schrödinger operator. If $G\left(z, x, x^{\prime}\right)$ denotes the Green's function of $H$ (as in (3.32), (3.33) ), formulas (3.47) - (3.49) for the resolvent of $H_{x_{0}}^{\beta}$ apply without change in the present general situation. In particular, defining

$$
\Gamma^{\beta}(z, x)= \begin{cases}\left(\beta+\partial_{1}\right)\left(\beta+\partial_{2}\right) G(z, x, x), & \beta \in \mathbb{R} \\ G(z, x, x), & \beta=\infty\end{cases}
$$

(cf. the notation introduction in $(3.39)$ ) one computes for $\beta \in \mathbb{R} \cup\{\infty\}$,

$$
\operatorname{Tr}\left[\left(H_{x}^{\beta}-z\right)^{-1}-(H-z)^{-1}\right]=-\frac{d}{d z} \ln \left[\Gamma^{\beta}(z, x)\right], z \in \mathbb{C} \backslash\left\{\sigma\left(H_{x}^{\beta}\right) \cup \sigma(H)\right\} .
$$

Given hypothesis (5.1), one can prove the existence of asymptotic expansions of the type

$$
\operatorname{Tr}\left[\left(H_{x}^{\beta}-z\right)^{-1}-(H-z)^{-1}\right] \underset{z \rightarrow i \infty}{=} \sum_{j=0}^{\infty} r_{j}^{\beta}(x) z^{-j}, \quad \beta \in \mathbb{R} \cup\{\infty\}
$$

uniformly with respect to $x \in \mathbb{R}$ (cf. [24]). In particular, one can derive the heat kernel expansion

$$
\operatorname{Tr}\left[e^{-\tau H_{x}^{\infty}}-e^{-\tau H}\right] \underset{\tau \downarrow 0}{\sim} \sum_{j=0}^{\infty} s_{j}^{\infty}(x) \tau^{j}, \quad x \in \mathbb{R},
$$

where

$$
s_{j}^{\infty}(x)=(-1)^{j+1}(j !)^{-1} r_{j}^{\infty}(x), \quad j \in \mathbb{N}_{0}
$$

and $s_{j}^{\infty}\left(r_{j}^{\infty}\right)$ are the well-known invariants of the KdV hierarchy.

In the special case of finite-gap potentials the connection of $\Gamma^{\beta}(z, s)$ in (5.4) with our polynomial approach in Section 3 is clearly demonstrated by 3.35 for $\beta=\infty$ and 3.53 . for $\beta=\mathbb{R}$. 
Before describing a new constructive (i.e., recursive) approach to the coefficients $r_{j}^{\beta}(x)$, $\beta \in \mathbb{R}$, we recall the definition of Krein's spectral shift function 30 associated with the pair $\left(H_{x}^{\beta}, H\right)$ (cf. [19], 23], [24]). The rank-one resolvent difference of $H_{x}^{\beta}$ and $H$ (cf. (3.47), $(3.48)$ is intimately connected with the fact that for each $x \in \mathbb{R}, \beta \in \mathbb{R} \cup\{\infty\}$,

$$
\Gamma^{\beta}(z, x) \text { is Herglotz with respect to } z
$$

(i.e., a holomorphic map $\mathbb{C}_{+} \rightarrow \mathbb{C}_{+}$, where $\mathbb{C}_{+}=\{z \in \mathbb{C} \mid \operatorname{Im}(z)>0\}$ ). The exponential Herglotz representation for $\Gamma^{\beta}(z, x)$ (cf. [3]) then reads for each $x \in \mathbb{R}$,

$$
\begin{array}{r}
\Gamma^{\beta}(z, x)=\exp \left\{c^{\beta}+\int_{\mathbb{R}}\left[(\lambda-z)^{-1}-\lambda\left(1+\lambda^{2}\right)^{-1}\right]\left[\xi^{\beta}(\lambda, x)+\delta^{\beta}\right] d \lambda\right\}, \\
c^{\beta} \in \mathbb{R}, \beta \in \mathbb{R} \cup\{\infty\}, \delta^{\beta}=\left\{\begin{array}{ll}
1, & \beta \in \mathbb{R} \\
0, & \beta=\infty
\end{array},\right.
\end{array}
$$

where, by Fatou's lemma,

$$
\left.\xi^{\beta}(\lambda, x)=\pi^{-1} \lim _{\epsilon \downarrow 0} \operatorname{Im}\left\{\ln \left[\beta+\partial_{1}\right)\left(\beta+\partial_{2}\right) G(\lambda+i \epsilon, x, x)\right]\right\}-\delta^{\beta}, \quad \beta \in \mathbb{R} \cup\{\infty\}
$$

for a.e. $\lambda \in \mathbb{R}$. Moreover,

$$
\begin{aligned}
& -1 \leq \xi^{\beta}(\lambda, x) \leq 0, \xi^{\beta}(\lambda, x)=0, \lambda<\inf \sigma\left(H_{x}^{\beta}\right), \beta \in \mathbb{R}, \\
& 0 \leq \xi^{\infty}(\lambda, x) \leq 1, \xi^{\infty}(\lambda, x)=0, \lambda<\inf \sigma(H)
\end{aligned}
$$

for a.e. $\lambda \in \mathbb{R}$. As a consequence, one obtains (cf. [39])

$$
\operatorname{Tr}\left[f\left(H_{x}^{\beta}\right)-f(H)\right]=\int_{\mathbb{R}} d \lambda f^{\prime}(\lambda) \xi^{\beta}(\lambda, x), \quad \beta \in \mathbb{R} \cup\{\infty\}, x \in \mathbb{R}
$$

for any $f \in C^{2}(\mathbb{R})$ with $\left(1+\lambda^{2}\right) f^{(j)} \in L^{2}((0, \infty)), j=1,2$ and for $f(\lambda)=(\lambda-z)^{-1}$, $z \in \mathbb{C} \backslash\left[\inf \sigma\left(H_{x}^{\beta}\right), \infty\right)$. In particular, (5.13) holds for traces of heat kernel and resolvent differences, i.e., for any $\beta \in \mathbb{R} \cup\{\infty\}, x \in \mathbb{R}$,

$$
\begin{aligned}
& \operatorname{Tr}\left[e^{-\tau H_{x}^{\beta}}-e^{-\tau H}\right]=-\tau \int_{e_{x, 0}^{\beta}}^{\infty} d \lambda e^{-\tau \lambda} \xi^{\beta}(\lambda, x), \quad \tau>0, \\
& \operatorname{Tr}\left[\left(H_{x}^{\beta}-z\right)^{-1}-(H-z)^{-1}\right]=-\int_{e_{x, 0}^{\beta}}^{\infty} d \lambda(\lambda-z)^{-2} \xi^{\beta}(\lambda, x), \quad z \in \mathbb{C} \backslash\left\{\sigma\left(H_{x}^{\beta}\right) \cup \sigma(H)\right\},
\end{aligned}
$$

where

$$
e_{x, 0}^{\beta}=\left\{\begin{array}{ll}
\inf \sigma\left(H_{x}^{\beta}\right), & \beta \in \mathbb{R} \\
\inf \sigma(H), & \beta=\infty
\end{array} .\right.
$$

Returning to a recursive approach for the expansion coefficients $r_{j}^{\beta}(x)$ in (5.6) we first consider the expansion

$$
\Gamma^{\beta}(z, x) \underset{z \rightarrow i \infty}{=}(i / 2) \sum_{j=-\delta^{\beta}}^{\infty} \gamma_{j}^{\beta}(x) z^{-j-1 / 2}, \quad \beta \in \mathbb{R} \cup\{\infty\} .
$$

(A comparison of (5.17) and 4.60 reveals that $\gamma_{j}^{\infty}(x)=\tilde{f}_{j}(x), j \in \mathbb{N}_{0}$ in the case $\beta=\infty$.) In order to obtain a recursion relation for $\gamma_{j}^{\beta}(x)$ one can use the following result. 
Lemma 5.1. Let $z \in \mathbb{C} \backslash \sigma(H), x \in \mathbb{R}$.

(i). Assume $\beta \in \mathbb{R}$. Then $\Gamma^{\beta}(z, x)=\left(\beta+\partial_{1}\right)\left(\beta+\partial_{2}\right) G(z, x, x)$ satisfies

$$
\begin{aligned}
& 2\left[V(x)-\beta^{2}-z\right] \Gamma_{x x}^{\beta}(z, x) \Gamma^{\beta}(z, x)-\left[V(x)-\beta^{2}-z\right] \Gamma_{x}^{\beta}(z, x)^{2}-2 V_{x}(x) \Gamma_{x}^{\beta}(z, x) \Gamma^{\beta}(z, x) \\
& -4\left\{[V(x)-z]\left[V(x)-\beta^{2}-z\right]-\beta V_{x}(x)\right\} \Gamma^{\beta}(z, x)^{2}=-\left[V(x)-z-\beta^{2}\right]^{3} .
\end{aligned}
$$

(ii). Assume $\beta=\infty$. Then $\Gamma^{\infty}(z, x)=G(z, x, x)$ satisfies

$$
\Gamma_{x x x}^{\infty}(z, x)-4[V(x)-z] \Gamma_{x}^{\infty}(z, x)-2 V_{x}(x) \Gamma^{\infty}(z, x)=0
$$

and

$$
-2 \Gamma_{x x}^{\infty}(z, x) \Gamma^{\infty}(z, x)+\Gamma_{x}^{\infty}(z, x)^{2}+4[V(x)-z] \Gamma^{\infty}(z, x)^{2}=1 .
$$

While the results (5.19) and (5.20) in the Dirichlet case $\beta=\infty$ are well-known, see, e.g., [14], the result (5.18) (with the exception of the Neumann case $\beta=0$ which was first presented in [21]) is new. Unfortunately, we have no reasonably short derivation of the differential equation (5.18). It can be verified (not without tears) after quite tedious though straightforward calculations (we recommend additional help in the form of symbolic computations).

Insertion of the expansion (5.17) into (5.18) and (5.20) in Lemma 5.1 yields

Lemma 5.2. The coefficients $\gamma_{j}^{\beta}(x)$ in (5.17) satisfy the following recursion relation.

(i). Assume $\beta \in \mathbb{R}$. Then

$$
\begin{aligned}
\gamma_{-1}^{\beta}= & 1, \gamma_{0}^{\beta}=\beta^{2}-\frac{1}{2} V, \gamma_{1}^{\beta}=\frac{1}{2} \beta^{2} V+\frac{1}{2} \beta V_{x}-\frac{1}{8} V^{2}+\frac{1}{8} V_{x x}, \\
\gamma_{2}^{\beta}= & -\frac{1}{16} V^{3}+\frac{3}{8} \beta^{2} V^{2}+\frac{3}{16} V_{x}\left(4 \beta V+V_{x}\right)+\frac{1}{8} V_{x x}\left(V-\beta^{2}\right)-\frac{1}{8} \beta V_{x x x}-\frac{1}{64} V_{x x x x}, \\
\gamma_{j+1}^{\beta}= & \frac{1}{8} \sum_{\ell=1}^{j}\left[2\left(V-\beta^{2}\right) \gamma_{\ell-1}^{\beta} \gamma_{j-\ell, x x}^{\beta}-\left(V-\beta^{2}\right) \gamma_{\ell-1, x}^{\beta} \gamma_{j-\ell, x}^{\beta}\right. \\
& \left.-4 \gamma_{\ell}^{\beta} \gamma_{j-\ell+1}^{\beta}-4 V\left(V-\beta^{2}\right) \gamma_{\ell-1}^{\beta} \gamma_{j-\ell}^{\beta}-2 V_{x} \gamma_{\ell-1}^{\beta} \gamma_{j-\ell, x}^{\beta}+\gamma_{\ell-1}^{\beta} \gamma_{j-\ell}^{\beta}\right] \\
& +\frac{1}{8} \sum_{\ell=0}^{j}\left[\gamma_{\ell, x}^{\beta} \gamma_{j-\ell, x}^{\beta}-2 \gamma_{\ell}^{\beta} \gamma_{j-\ell, x x}^{\beta}-4\left(\beta^{2}-2 V\right) \gamma_{\ell}^{\beta} \gamma_{j-\ell}^{\beta}\right], \quad j \geq 2 .
\end{aligned}
$$

(ii). Assume $\beta=\infty$. Then

$$
\begin{aligned}
\gamma_{0}^{\infty} & =1, \gamma_{1}^{\infty}=\frac{1}{2} V, \\
\gamma_{j+1}^{\infty} & =-\frac{1}{2} \sum_{\ell=1}^{j} \gamma_{\ell}^{\infty} \gamma_{j+1-\ell}^{\infty}+\frac{1}{2} \sum_{\ell=0}^{j}\left[V \gamma_{\ell}^{\infty} \gamma_{j-\ell}^{\infty}+\frac{1}{4} \gamma_{\ell, x}^{\infty} \gamma_{j-\ell, x}^{\infty}-\frac{1}{2} \gamma_{\ell, x x}^{\infty} \gamma_{j-\ell}^{\infty}\right], \quad j \geq 1 .
\end{aligned}
$$

The final result for $r_{j}^{\beta}(x)$ then reads

Theorem 5.3. The coefficients $r_{j}^{\beta}(x)$ in (5.6) satisfy the following recursion relations. (i). Assume $\beta \in \mathbb{R}$. Then

$$
r_{0}^{\beta}(x)=-\frac{1}{2}, r_{1}^{\beta}(x)=\beta^{2}-\frac{1}{2} V(x), r_{j}^{\beta}(x)=j \gamma_{j-1}^{\beta}(x)-\sum_{\ell=1}^{j-1} \gamma_{j-\ell-1}^{\beta}(x) r_{\ell}^{\beta}(x), \quad j \geq 2 .
$$

(ii). Assume $\beta=\infty$. Then

$$
r_{0}^{\infty}=\frac{1}{2}, r_{1}^{\infty}(x)=\frac{1}{2} V(x), r_{j}^{\infty}(x)=j \gamma_{j}^{\infty}(x)-\sum_{\ell=1}^{j-1} \gamma_{j-\ell}^{\infty}(x) r_{\ell}^{\infty}(x), \quad j \geq 2 .
$$

Proof. It suffices to combine (5.5), (5.6), (5.17), and the following well-known fact on asymptotic expansions: $F(z) \underset{|z| \rightarrow \infty}{=} \sum_{j=1}^{\infty} c_{j} z^{-j}$ implies $\ln [1+F(z)] \underset{|z| \rightarrow \infty}{=} \sum_{j=1}^{\infty} d_{j} z^{-j}$, where $d_{1}=c_{1}, d_{j}=c_{j}-\sum_{\ell=1}^{j-1}(\ell / j) c_{j-\ell} d_{\ell}, j \geq 2$. 
Theorem 5.3 (i) has first been derived (by using a different strategy) in [24]. The current derivation, based on the universal differential equation (5.18), is new. Combined with (5.21), Theorem 5.3 (i) yields the most efficient algorithm to date for computing $r_{j}^{\beta}(x), \beta \in \mathbb{R}$.

The connection between $r_{j}^{\beta}(x)$ and $\xi^{\beta}(\lambda, x)$ is illustrated in the following result.

Theorem 5.4. [24] Let $e_{x, 0}^{\beta}=\inf \sigma\left(H_{x}^{\beta}\right), \beta \in \mathbb{R}, e_{0}^{\infty}=\inf \sigma(H)$.

(i). Assume $\beta \in \mathbb{R}$. Then

$$
r_{j}^{\beta}(x)=-\frac{1}{2}\left(e_{x, 0}^{\beta}\right)^{j}-\lim _{z \rightarrow i \infty} \int_{e_{x, 0}^{\beta}}^{\infty} d \lambda z^{j+1}(\lambda-z)^{-j-1} j(-\lambda)^{j-1}\left[\frac{1}{2}+\xi^{\beta}(\lambda, x)\right], j \in \mathbb{N} .
$$

(ii). Assume $\beta=\infty$. Then

$$
r_{j}^{\infty}(x)=\frac{1}{2}\left(e_{0}^{\infty}\right)^{j}+\lim _{z \rightarrow i \infty} \int_{e_{0}^{\infty}}^{\infty} d \lambda z^{j+1}(\lambda-z)^{-j-1} j(-\lambda)^{j-1}\left[\frac{1}{2}-\xi^{\infty}(\lambda, x)\right], j \in \mathbb{N} .
$$

We conclude with an example that yields the higher-order trace formulas for periodic potentials which also applies to the (quasi-periodic) finite-gap potentials of Section 3.

Example 5.5. Assume $V$ is periodic, i.e., for some $\Omega>0, V(x+\Omega)=V(x)$ for all $x \in \mathbb{R}$ in addition to (5.1). Then Floquet theory implies

$$
\sigma(H)=\bigcup_{n=1}^{\infty}\left[E_{2(n-1)}, E_{2 n-1}\right], E_{0}<E_{1} \leq E_{2}<E_{3} \leq \cdots
$$

(i). Assume $\beta \in \mathbb{R}$. Then

$$
\begin{gathered}
\sigma\left(H_{x}^{\beta}\right)=\left\{\lambda_{n}^{\beta}(x)\right\}_{n \in \mathbb{N}_{0}} \cup \sigma(H), \lambda_{0}^{\beta}(x) \leq E_{0}, \lambda_{n}^{\beta}(x) \in\left[E_{2 n-1}, E_{2 n}\right], \quad n \in \mathbb{N}, \\
\xi^{\beta}(\lambda, x)= \begin{cases}0, & \lambda<\lambda_{0}^{\beta}(x), E_{2 n-1}<\lambda<\lambda_{n}^{\beta}(x), \quad n \in \mathbb{N} \\
-1, & \lambda_{0}^{\beta}(x)<\lambda<E_{0}, \quad \lambda_{n}^{\beta}(x)<\lambda<E_{2 n}, \quad n \in \mathbb{N} . \\
-\frac{1}{2}, & E_{2(n-1)}<\lambda<E_{2 n-1}, \quad n \in \mathbb{N}\end{cases}
\end{gathered}
$$

Inserting (5.29) into (5.25) then yields the higher-order periodic trace formulas

$$
r_{j}^{\beta}(x)=\frac{1}{2} E_{0}^{j}-\lambda_{0}^{\beta}(x)^{j}+\frac{1}{2} \sum_{n=1}^{\infty}\left[E_{2 n-1}^{j}+E_{2 n}^{j}-2 \lambda_{n}^{\beta}(x)^{j}\right], \quad j \in \mathbb{N} .
$$

(ii). Assume $\beta=\infty$. Then

$$
\begin{gathered}
\sigma\left(H_{x}^{\infty}\right)=\left\{\mu_{n}(x)\right\}_{n \in \mathbb{N}} \cup \sigma(H), \mu_{n}(x) \in\left[E_{2 n-1}, E_{2 n}\right], \quad n \in \mathbb{N}, \\
\xi^{\infty}(\lambda, x)= \begin{cases}0, & \lambda<E_{0}, \mu_{n}(x)<\lambda<E_{2 n}, \quad n \in \mathbb{N} \\
1, & E_{2 n-1}<\lambda<\mu_{n}(x), \quad n \in \mathbb{N} \\
\frac{1}{2}, & E_{2(n-1)}<\lambda<E_{2 n-1}, \quad n \in \mathbb{N} .\end{cases}
\end{gathered}
$$

Insertion of 5.32 into 5.26 then yields

$$
r_{j}^{\infty}(x)=\frac{1}{2} E_{0}^{j}+\frac{1}{2} \sum_{n=1}^{\infty}\left[E_{2 n-1}^{j}+E_{2 n}^{j}-2 \mu_{n}(x)^{j}\right], \quad j \in \mathbb{N} .
$$

The results (5.29) and (5.32) remain valid in the algebro-geometric finite-gap situation discussed in Section 3 where

$$
E_{2 n+1}=\lambda_{n}^{\beta}(x)=E_{2 n+2}, \quad n \geq g+1, \beta \in \mathbb{R} \cup\{\infty\} .
$$

Hence (5.30) and (5.33) apply to the stationary KdV solutions of Section 3 (e.g., (5.33) for $j=1$ coincides with (3.19) $)$ which, in general, are quasi-periodic with respect to $x$. Moreover, (5.30) and (5.33) also extend to certain classes of almost-periodic $V(x)$, see, e.g., [8], [28], [31], [32], Chs. 9, 11. 
The periodic Dirichlet trace formula (5.33) for $j=1$ has been noticed by Hochstadt [25] and later on by Dubrovin [10]. The general case $j=\mathbb{N}$ appeared in McKean and van Moerbeke [34] and Flaschka [13]. More recent accounts of (5.33) can be found in [8], [28], [31], [32], Chs. 9, 11, [40]. The Neumann case $\beta=0$ in (5.30) is due to McKean and Trubowitz 35]. The general case $\beta \in \mathbb{R}$ was first studied in [24]. Additional references on the subject of trace formulas and their use in connection with the inverse spectral problem can be found in the papers cited in this paragraph and in the ones listed at the beginning of this section.

\section{Appendix A. Hyperelliptic Curves of the KdV-type and Theta Functions}

We briefly summarize our basic notation for hyperelliptic KdV-type curves (i.e., those branched at infinity) and their theta functions as employed in Sections 3 and 4 . For details on this standard material we refer, e.g., to [11], [12, [29], 37].

Consider the points $\left\{E_{n}\right\}_{0 \leq n \leq g} \subset \mathbb{R}, E_{0}<E_{1}<\cdots<E_{2 g}, g \in \mathbb{N}_{0}$ and define the cut plane $\Pi=\mathbb{C} \backslash \bigcup_{j=0}^{g-1}\left[E_{2 j}, E_{2 j+1}\right] \cup\left[E_{2 g}, \infty\right)$ with the holomorphic function

$$
R_{2 g+1}(.)^{1 / 2}:\left\{\begin{array}{l}
\Pi \rightarrow \mathbb{C} \\
z \rightarrow\left[\prod_{n=0}^{2 g}\left(z-E_{n}\right)\right]^{1 / 2}
\end{array}\right.
$$

on it. $R_{2 g+1}(.)^{1 / 2}$ is extended to all of $\mathbb{C}$ by

$$
R_{2 g+1}(\lambda)^{1 / 2}=\lim _{\epsilon \downarrow 0} R_{2 g+1}(\lambda+i \epsilon)^{1 / 2}, \quad \lambda \in \mathbb{C} \backslash \Pi,
$$

with the sign of the square root chosen according to

$$
R_{2 g+1}(\lambda)^{1 / 2}=\left\{\begin{array}{lll}
(-1)^{g} i\left|R_{2 g+1}(\lambda)^{1 / 2}\right|, & \lambda \in\left(-\infty, E_{0}\right) & \\
(-1)^{g+j} i\left|R_{2 g+1}(\lambda)^{1 / 2}\right|, & \lambda \in\left(E_{2 j-1}, E_{2 j}\right), & 1 \leq j \leq g \\
(-1)^{g+j}\left|R_{2 g+1}(\lambda)^{1 / 2}\right|, & \lambda \in\left(E_{2 j}, E_{2 j+1}\right), & 0 \leq j \leq g-1 \\
\left|R_{2 g+1}(\lambda)^{1 / 2}\right|, & \lambda \in\left(E_{2 g}, \infty\right) &
\end{array} .\right.
$$

Next we define the set

$$
M=\left\{\left(z, \sigma R_{2 g+1}(z)^{1 / 2}\right) \mid z \in \mathbb{C}, \sigma \in\{-,+\}\right\} \cup\left\{P_{\infty}=(\infty, \infty)\right\}
$$

and

$$
B=\left\{\left(E_{n}, 0\right)\right\}_{0 \leq n \leq 2 g} \cup\left\{P_{\infty}=(\infty, \infty)\right\},
$$

the set of branch points. $M$ becomes a compact Riemann surface upon introducing the charts $\left(U_{P_{0}}, \zeta_{P_{0}}\right)$ defined as follows

$$
\begin{aligned}
P_{0} & =\left(z_{0}, \sigma_{0} R_{2 g+1}(z)^{1 / 2}\right) \text { or } P_{0}=P_{\infty}, \\
P & =\left(z, \sigma R_{2 g+1}(z)^{1 / 2}\right) \in U_{P_{0}} \subset M, V_{P_{0}}=\zeta\left(U_{P_{0}}\right) \subset \mathbb{C} .
\end{aligned}
$$

$\boldsymbol{P}_{\mathbf{0}} \in \boldsymbol{M \backslash B}$

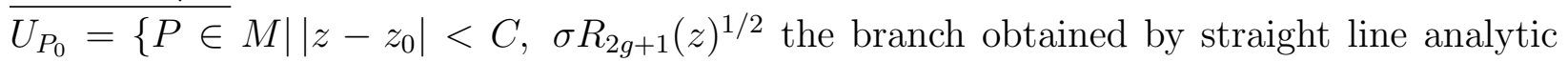
continuation starting from $\left.z_{0}\right\}, C=\min _{n}\left|z_{0}-E_{n}\right|$,

$$
\zeta_{P_{0}}:\left\{\begin{array}{l}
U_{P_{0}} \rightarrow V_{P_{0}} \\
P \rightarrow\left(z-z_{0}\right)
\end{array} \quad, \quad \zeta_{P_{0}}^{-1}:\left\{\begin{array}{l}
V_{P_{0}} \rightarrow U_{P_{0}} \\
\zeta \rightarrow\left(z_{0}+\zeta, \sigma R_{2 g+1}\left(z_{0}+\zeta\right)^{1 / 2}\right)
\end{array}\right.\right.
$$




$$
\begin{aligned}
& \underline{P_{0}=\left(E_{n_{0}}, 0\right):} \\
& U_{P_{0}}=\left\{P \in M|| z-E_{n_{0}} \mid<C_{n_{0}}\right\}, \quad C_{n_{0}}=\left\{\begin{array}{ll}
\min _{n \neq n_{0}}\left|E_{n}-E_{n_{0}}\right|, & g \in \mathbb{N} \\
\infty, & g=0
\end{array},\right. \\
& V_{P_{0}}=\left\{\zeta \in \mathbb{C}|| \zeta \mid<C_{n_{0}}^{1 / 2}\right\}, \quad \zeta_{P_{0}}:\left\{\begin{array}{l}
U_{P_{0}} \rightarrow V_{P_{0}} \\
P \rightarrow \sigma\left(z-E_{n_{0}}\right)^{1 / 2}
\end{array}\right. \\
& \left(z-E_{n_{0}}\right)^{1 / 2}=\left|\left(z-E_{n_{0}}\right)^{1 / 2}\right| e^{(i / 2) \arg \left(z-E_{n_{0}}\right)}, \arg \left(z-E_{n_{0}}\right) \in\left\{\begin{array}{ll}
{[0,2 \pi),} & n_{0} \text { even } \\
(-\pi, \pi], & n_{0} \text { odd }
\end{array},\right. \\
& \zeta_{P_{0}}^{-1}:\left\{\begin{array}{l}
V_{P_{0}} \rightarrow U_{P_{0}} \\
\zeta \rightarrow\left(E_{n_{0}}+\zeta^{2}, \zeta\left[\prod_{n \neq n_{0}}\left(E_{n_{0}}-E_{n}+\zeta^{2}\right)\right]^{1 / 2}\right.
\end{array}\right. \\
& {\left[\prod_{n \neq n_{0}}\left(E_{n_{0}}-E_{n}+\zeta^{2}\right)\right]^{1 / 2}=(-1)^{g} i^{-n_{0}}\left|\left[\prod_{n \neq n_{0}}\left(E_{n_{0}}-E_{n}\right)\right]^{1 / 2}\right|\left[1+2^{-1} \zeta^{2} \sum_{n \neq n_{0}}\left(E_{n_{0}}-E_{n}\right)^{-1}+0\left(\zeta^{4}\right)\right] .}
\end{aligned}
$$

$$
\begin{aligned}
& \frac{\boldsymbol{P}_{\mathbf{0}}=\boldsymbol{P}_{\infty}:}{U_{P_{0}}=}=\left\{P \in M|| z \mid>C_{\infty}\right\}, C_{\infty}=\max _{n}\left|E_{n}\right|, V_{P_{0}}=\left\{\zeta \in \mathbb{C}|| \zeta \mid<C_{\infty}^{-1 / 2}\right\}, \\
& \zeta_{P_{0}}:\left\{\begin{array}{l}
U_{P_{0}} \rightarrow V_{P_{0}} \\
P \rightarrow \sigma\left(1 / z^{1 / 2}\right), \quad z^{1 / 2}=\left|z^{1 / 2}\right| e^{(i / 2) \arg (z)}, \\
P_{\infty} \rightarrow 0
\end{array}\right. \\
& \zeta_{P_{0}}^{-1}:\left\{\begin{array}{l}
V_{P_{0}} \rightarrow U_{P_{0}} \\
\zeta \rightarrow\left(\zeta^{-2}, \zeta^{-2 g-1}\left[\prod_{n}\left(1-\zeta^{2} E_{n}\right)\right]^{1 / 2}\right), \\
0 \rightarrow P_{\infty}
\end{array}\right. \\
& {\left[\prod_{n}\left(1-\zeta^{2} E_{n}\right)\right]^{1 / 2}=1-2^{-1} \zeta^{2} \sum_{n} E_{n}+0\left(\zeta^{4}\right) . }
\end{aligned}
$$

Upper and lower sheets $\Pi_{ \pm} \subset M$ with associated charts $\zeta_{ \pm}$are defined by

$$
\Pi_{ \pm}=\left\{\left(z, \pm R_{2 g+1}(z)^{1 / 2}\right) \in M \mid z \in \Pi\right\}, \zeta_{ \pm}:\left\{\begin{array}{l}
\Pi_{ \pm} \rightarrow \Pi \\
P \rightarrow z
\end{array} .\right.
$$

The compact Riemann surface (curve) resulting from A.4 - A.9 is denoted by $K_{g}$. Topologically, $K_{g}$ is a sphere with $g$ handles and hence has genus $g$.

Next, define the holomorphic sheet exchange map (involution)

$$
*:\left\{\begin{array}{l}
K_{g} \rightarrow K_{g} \\
\left(z, \sigma R_{2 g+1}(z)^{1 / 2}\right) \rightarrow\left(z, \sigma R_{2 g+1}(z)^{1 / 2}\right)^{*}=\left(z,-\sigma R_{2 g+1}(z)^{1 / 2}\right)
\end{array}\right.
$$

and the two meromorphic projection maps

$$
\tilde{\pi}:\left\{\begin{array}{l}
K_{g} \rightarrow \mathbb{C} \cup\{\infty\} \\
\left(z, \sigma R_{2 g+1}(z)^{1 / 2}\right) \rightarrow z \\
P_{\infty} \rightarrow \infty
\end{array} \quad, \quad R_{2 g+1}^{1 / 2}:\left\{\begin{array}{l}
K_{g} \rightarrow \mathbb{C} \cup\{\infty\} \\
\left(z, \sigma R_{2 g+1}(z)^{1 / 2}\right) \rightarrow \sigma R_{2 g+1}(z)^{1 / 2} \\
P_{\infty} \rightarrow \infty
\end{array}\right.\right.
$$


$\tilde{\pi}$ has a pole of order 2 at $P_{\infty}$ and two simple zeros at $\left(0, \pm R_{2 g+1}(0)^{1 / 2}\right)$ if $R_{2 g+1}(0) \neq 0$ or a double zero at $(0,0)$ if $R_{2 g+1}(0)=0$ (i.e., if $0 \in\left\{E_{n}\right\}_{0 \leq n \leq 2 g}$ ) and $R_{2 g+1}^{1 / 2}$ has a pole of order $2 g+1$ at $P_{\infty}$ and $2 g+1$ simple zeros at $\left(E_{n}, 0\right), 0 \leq n \leq 2 g$. Moreover,

$$
\tilde{\pi}\left(P^{*}\right)=\tilde{\pi}(P), R_{2 g+1}^{1 / 2}\left(P^{*}\right)=-R_{2 g+1}^{1 / 2}(P), \quad P \in K_{g} .
$$

Thus $K_{g}$ is a two-sheeted ramified covering of the Riemann sphere $\mathbb{C}_{\infty}(\cong \mathbb{C} \cup\{\infty\})$, in particular, $K_{g}$ is compact and hyperelliptic.

Using our local charts one infers that for $g \in \mathbb{N}, d \tilde{\pi} / R_{2 g+1}^{1 / 2}$ is a holomorphic differential on $K_{g}$ with a zero of order $2(g-1)$ at $P_{\infty}$ and hence

$$
\eta_{j}=\tilde{\pi}^{j-1} d \tilde{\pi} / R_{2 g+1}^{1 / 2}(.), \quad 1 \leq j \leq g
$$

form a basis for the space of holomorphic differentials on $K_{g}$.

Next we introduce a canonical homology basis $\left\{a_{j}, b_{j}\right\}_{1 \leq j \leq g}$ for $K_{g}$ as follows. The cycle $a_{\ell}$ starts near $E_{2 \ell-1}$ on $\Pi_{+}$, surrounds $E_{2 \ell}$ counterclockwise thereby changing to $\Pi_{-}$, and returns to the starting point encircling $E_{2 \ell-1}$, changing sheets again. The cycle $b_{\ell}$ surrounds $E_{0}, E_{2 \ell-1}$ counterclockwise (once) on $\Pi_{+}$. The cycles are chosen such that their intersection matrix reads $a_{j} \circ b_{k}=\delta_{j, k}, 1 \leq j, k \leq g$. Introducing the invertible matrix $C$ in $\mathbb{C}^{g}$,

$$
\begin{aligned}
C & =\left(C_{j, k}\right)_{1 \leq j, k \leq g}, C_{j, k}=\int_{a_{k}} \eta_{j}=2 \int_{E_{2 k-1}}^{E_{2 k}} z^{j-1} d z / R_{2 g+1}(z)^{1 / 2} \in i \mathbb{R}, \\
\underline{c}(k) & =\left(c_{1}(k), \ldots, c_{g}(k)\right), \quad c_{j}(k)=C_{j, k}^{-1},
\end{aligned}
$$

the normalized differentials $\omega_{j}, 1 \leq j \leq g$,

$$
\omega_{j}=\sum_{\ell=1}^{g} c_{j}(\ell) \eta_{\ell}, \int_{a_{k}} \omega_{j}=\delta_{j, k}, \quad 1 \leq j, k \leq g
$$

form a canonical basis for the space of holomorphic differentials on $K_{g}$. The matrix $\tau$ in $\mathbb{C}^{g}$ of $b$-periods,

$$
\tau=\left(\tau_{j, k}\right)_{1 \leq j, k \leq g}, \tau_{j, k}=\int_{b_{k}} \omega_{j}, \quad 1 \leq j, k \leq g
$$

then satisfies

$$
\tau_{j, k}=\tau_{k, j}, \quad 1 \leq j, k \leq g, \tau=i T, \quad T>0 .
$$

In the chart $\left(U_{P_{\infty}}, \zeta_{P_{\infty}}=\zeta\right)$ induced by $1 / \tilde{\pi}^{1 / 2}$ near $P_{\infty}$ one infers

$$
\begin{aligned}
\underline{\omega} & =-2\left\{\sum_{j=1}^{g} \underline{c}(j) \zeta^{2(g-j)} /\left[\prod_{n}\left(1-\zeta^{2} E_{n}\right)\right]^{1 / 2}\right\} d \zeta \\
& =-2\left\{\underline{c}(g)+\left[\frac{1}{2} \underline{c}(g) \sum_{n=0}^{2 g} E_{n}+\underline{c}(g-1)\right] \zeta^{2}+0\left(\zeta^{4}\right)\right\} d \zeta .
\end{aligned}
$$

Associated with the homology basis $\left\{a_{j}, b_{j}\right\}_{1 \leq j \leq g}$ we also recall the canonical dissection of $K_{g}$ along its cycles yielding the simply connected interior $\hat{K}_{g}$ of the fundamental polygon $\partial \hat{K}_{g}$ given by $\partial \hat{K}_{g}=a_{1} b_{1} a_{1}^{-1} b_{1}^{-1} a_{2} b_{2} a_{2}^{-1} b_{2}^{-1} \cdots a_{g}^{-1} b_{g}^{-1}$. The Riemann theta function associated with $K_{g}$ is defined by

$$
\theta(\underline{z})=\sum_{\underline{n} \in \mathbb{Z}^{g}} \exp [2 \pi i(\underline{n}, \underline{z})+\pi i(\underline{n}, \tau \underline{n})], \quad \underline{z}=\left(z_{1}, \ldots, z_{g}\right) \in \mathbb{C}^{g},
$$


where $(\underline{u}, \underline{v})=\sum_{j=1}^{g} \overline{u_{j}} v_{j}$ denotes the scalar product in $\mathbb{C}^{g}$. It has the fundamental properties

$$
\begin{aligned}
& \theta\left(z_{1}, \ldots, z_{j-1},-z_{j}, z_{j+1}, \ldots, z_{g}\right)=\theta(\underline{z}), \\
& \theta(\underline{z}+\underline{m}+\tau \underline{n})=\theta(\underline{z}) \exp [-2 \pi i(\underline{n}, \underline{z})-\pi i(\underline{n}, \tau \underline{n})], \quad \underline{m}, \underline{n} \in \mathbb{Z}^{g} .
\end{aligned}
$$

A divisor $\mathcal{D}$ on $K_{g}$ is a map $\mathcal{D}: K_{g} \rightarrow \mathbb{Z}$, where $\mathcal{D}(P) \neq 0$ for only finitely-many $P \in K_{g}$. The set of all divisors will be denoted by $\operatorname{Div}\left(K_{g}\right)$. With $L_{g}$ we denote the period lattice

$$
L_{g}=\left\{\underline{z} \in \mathbb{C}^{g} \mid \underline{z}=\underline{m}+\tau \underline{n}, \underline{m}, \underline{n} \in \mathbb{Z}^{g}\right\}
$$

and the Jacobi variety $J\left(K_{g}\right)$ is defined by

$$
J\left(K_{g}\right)=\mathbb{C}^{g} / L_{g}
$$

The Abel maps $\underline{A}_{P_{0}}($.$) , respectively \underline{\alpha}_{P_{0}}($.$) are defined by$

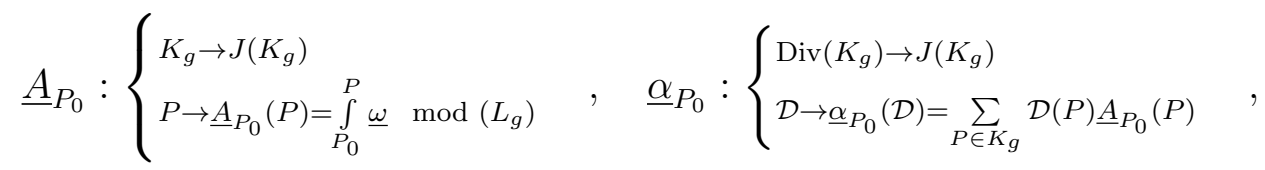

with $P_{0} \in K_{g}$ a fixed base point. (In the main text we agree to fix $P_{0}=\left(E_{0}, 0\right)$ for convenience.)

Next, let $\mathcal{M}\left(K_{g}\right)$ and $\mathcal{M}^{1}\left(K_{g}\right)$ denote the set of meromorphic functions (0-forms) and meromorphic differentials (1-forms) on $K_{g}$. The residue of a meromorphic differential $\nu \in$ $\mathcal{M}^{1}\left(K_{g}\right)$ at a point $Q_{0} \in K_{g}$ is defined by $\operatorname{res}_{Q_{0}}(\nu)=(2 \pi i)^{-1} \int_{\gamma_{Q_{0}}} \nu$, where $\gamma_{Q_{0}}$ is a counterclockwise oriented smooth simple closed contour encircling $Q_{0}$ but no other pole of $\nu$. Holomorphic differentials are also called (Abelian) differentials of the first kind (dfk); (Abelian) differentials of the second kind (dsk) $\omega^{(2)} \in \mathcal{M}^{1}\left(K_{g}\right)$ are characterized by the property that all their residues vanish. They are normalized, e.g., by demanding that all their $a$-periods vanish, i.e.,

$$
\int_{a_{j}} \omega^{(2)}=0, \quad 1 \leq j \leq g .
$$

If $\omega_{P_{1}, n}^{(2)}$ is a dsk on $K_{g}$ whose only pole is $P_{1} \in \hat{K}_{g}$ with principal part $\zeta^{-n-2} d \zeta, n \in \mathbb{N}_{0}$ near $P_{1}$ and $\omega_{j}=\left(\sum_{m=0}^{\infty} d_{j, m}\left(P_{1}\right) \zeta^{m}\right) d \zeta$ near $P_{1}$, then

$$
\int_{b_{j}} \omega_{P_{1, n}}^{(2)}=[2 \pi i /(n+1)] d_{j, n}\left(P_{1}\right) .
$$

A basis for dsk's on $K_{g}$, holomorphic on $K_{g} \backslash\left\{P_{\infty}\right\}$, is provided by

$$
\omega_{n}^{(2)}=\tilde{\pi}^{g+1+n} d \tilde{\pi} / R_{2 g+1}^{1 / 2}(.), \quad n \in \mathbb{N}_{0} .
$$

Any meromorphic differential $\omega^{(3)}$ on $K_{g}$ not of the first or second kind is defined to be of the third kind (dtk). A dtk $\omega^{(3)} \in \mathcal{M}^{1}\left(K_{g}\right)$ is usually normalized by the vanishing of its $a$-periods, i.e.,

$$
\int_{a_{j}} \omega^{(3)}=0, \quad 1 \leq j \leq g
$$

A normal dtk $\omega_{P_{1}, P_{2}}^{(3)}$ associated with two points $P_{1}, P_{2} \in \hat{K}_{g}, P_{1} \neq P_{2}$ by definition has simple poles at $P_{1}$ and $P_{2}$ with residues +1 at $P_{1}$ and -1 at $P_{2}$ and vanishing $a$-periods. If 
$\omega_{P, Q}^{(3)}$ is a normal dtk associated with $P, Q \in \hat{K}_{g}$, holomorphic on $K_{g} \backslash\{P, Q\}$, then

$$
\int_{b_{j}} \omega_{P, Q}^{(3)}=2 \pi i \int_{Q}^{P} \omega_{j}, \quad 1 \leq j \leq g,
$$

where the path from $Q$ to $P$ lies in $\hat{K}_{g}$ (i.e., does not touch any of the cycles $a_{j}, b_{j}$ ).

We shall always assume (without loss of generality) that all poles of dsk's and dtk's on $K_{g}$ lie on $\hat{K}_{g}$ (i.e., not on $\partial \hat{K}_{g}$ ).

For $f \in \mathcal{M}\left(K_{g}\right) \backslash\{0\}, \omega \in \mathcal{M}^{1}\left(K_{g}\right) \backslash\{0\}$ the divisors of $f$ and $\omega$ are denoted by $(f)$ and $(\omega)$, respectively. Two divisors $\mathcal{D}, \mathcal{E} \in \operatorname{Div}\left(K_{g}\right)$ are called equivalent, denoted by $\mathcal{D} \sim \mathcal{E}$, if and only if $\mathcal{D}-\mathcal{E}=(f)$ for some $f \in \mathcal{M}\left(K_{g}\right) \backslash\{0\}$. The divisor class $[\mathcal{D}]$ of $\mathcal{D}$ is then given by $[\mathcal{D}]=\left\{\mathcal{E} \in \operatorname{Div}\left(K_{g}\right) \mid \mathcal{E} \sim \mathcal{D}\right\}$. We recall that

$$
\operatorname{deg}((f))=0, \operatorname{deg}((\omega))=2(g-1), \quad f \in \mathcal{M}\left(K_{g}\right) \backslash\{0\}, \omega \in \mathcal{M}^{1}\left(K_{g}\right) \backslash\{0\},
$$

where the degree $\operatorname{deg}(\mathcal{D})$ of $\mathcal{D}$ is given by $\operatorname{deg}(\mathcal{D})=\sum_{P \in K_{g}} \mathcal{D}(P)$. One calls $(f)$ (respectively $(\omega))$ a principal (respectively canonical) divisor.

Introducing the complex linear spaces

$$
\begin{aligned}
\mathcal{L}(\mathcal{D}) & =\left\{f \in \mathcal{M}\left(K_{g}\right) \mid f=0 \text { or }(f) \geq \mathcal{D}\right\}, r(\mathcal{D})=\operatorname{dim}_{\mathbb{C}} \mathcal{L}(\mathcal{D}), \\
\mathcal{L}^{1}(\mathcal{D}) & =\left\{\omega \in \mathcal{M}^{1}\left(K_{g}\right) \mid \omega=0 \text { or }(\omega) \geq \mathcal{D}\right\}, i(\mathcal{D})=\operatorname{dim}_{\mathbb{C}} \mathcal{L}^{1}(\mathcal{D})
\end{aligned}
$$

$(i(\mathcal{D})$ the index of speciality of $\mathcal{D})$, one infers that $\operatorname{deg}(\mathcal{D}), r(\mathcal{D})$, and $i(\mathcal{D})$ only depend on the divisor class $[\mathcal{D}]$ of $\mathcal{D}$. Moreover, we recall the following fundamental facts.

Theorem A.1. Let $\mathcal{D} \in \operatorname{Div}\left(K_{g}\right), \omega \in \mathcal{M}^{1}\left(K_{g}\right) \backslash\{0\}$. Then

$$
i(\mathcal{D})=r(\mathcal{D}-(\omega)), \quad g \in \mathbb{N}_{0} .
$$

(ii). (Riemann-Roch theorem).

$$
r(-\mathcal{D})=\operatorname{deg}(\mathcal{D})+i(\mathcal{D})-g+1, \quad g \in \mathbb{N}_{0}
$$

(iii). (Abel's theorem). $\mathcal{D} \in \operatorname{Div}\left(K_{g}\right), g \in \mathbb{N}$ is principal if and only if

$$
\operatorname{deg}(\mathcal{D})=0 \text { and } \underline{\alpha}_{P_{0}}(\mathcal{D})=\underline{0} .
$$

(iv). (Jacobi's inversion theorem). Assume $g \in \mathbb{N}$, then $\underline{\alpha}_{P_{0}}: \operatorname{Div}\left(K_{g}\right) \rightarrow J\left(K_{g}\right)$ is surjective.

For notational convenience we agree to abbreviate

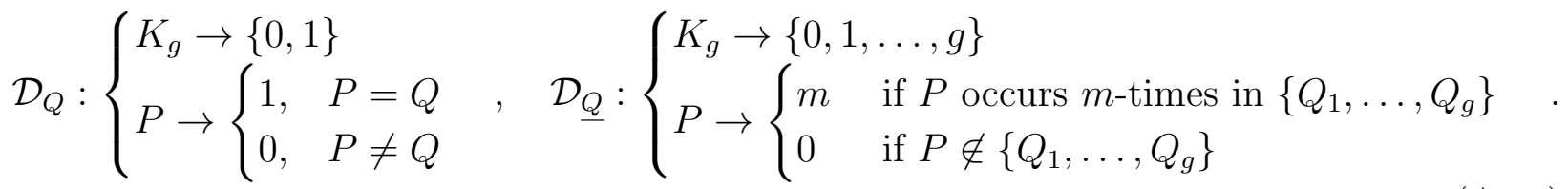

for $\underline{Q}=\left(Q_{1}, \ldots, Q_{g}\right) \in \sigma^{g} K_{g}\left(\sigma^{n} K_{g}\right.$ then $n$-th symmetric power of $\left.K_{g}\right)$. Moreover, $\sigma^{n} K_{g}$ can be identified with the set of positive divisors $0<\mathcal{D} \in \operatorname{Div}\left(K_{g}\right)$ of degree $n$.

Lemma A.2. Let $\mathcal{D}_{Q} \in \sigma^{g} K_{g}, \underline{Q}=\left(Q_{1}, \ldots, Q_{g}\right)$. Then $1 \leq i\left(\mathcal{D}_{Q}\right)=s(\leq g / 2)$ if and only if there are $s$ pairs of the type $\left(\overline{P, P^{*}}\right) \in\left\{Q_{1}, \ldots, Q_{g}\right\}$ (this includes, of course, branch points for which $P=P^{*}$ ). 
We emphasize that most results in this appendix immediately extend to the case where $\left\{E_{n}\right\}_{0 \leq n \leq 2 g} \subset \mathbb{C}$. (In this case $\tau$ is no longer purely imaginary as stated in A.18 but has a positive definite imaginary part.)

\section{Appendix B. An Explicit Illustration of the Riemann-Roch Theorem}

Finally, we give a brief illustration of the Riemann-Roch theorem in connection with KdV-type hyperelliptic curves, i.e., hyperelliptic curves branched at infinity, and explicitly determine a basis for the vector space $\mathcal{L}\left(-n \mathcal{D}_{P_{\infty}}-\mathcal{D}_{\hat{\mu}\left(x_{0}\right)}\right), n \in \mathbb{N}_{0}$.

We freely use the notation introduced in Appendix $A$ and refer, in particular, to the definition A.31 of $\mathcal{L}(\mathcal{D})$ and the Riemann-Roch theorem stated in Theorem A.1 (ii). In addition, we use the short-hand notation

$$
n \mathcal{D}_{P_{\infty}}+\mathcal{D}_{\underline{\hat{\mu}}\left(x_{0}\right)}=\sum_{m=1}^{n} \mathcal{D}_{P_{\infty}}+\sum_{j=1}^{g} \mathcal{D}_{\hat{\mu}_{j}\left(x_{0}\right)}, \quad n \in \mathbb{N}_{0}, \underline{\hat{\mu}}\left(x_{0}\right)=\left(\hat{\mu}_{1}\left(x_{0}\right), \ldots, \hat{\mu}_{g}\left(x_{0}\right)\right)
$$

and recall that

$$
\mathcal{L}\left(-n \mathcal{D}_{P_{\infty}}-\mathcal{D}_{\underline{\hat{\mu}}\left(x_{0}\right)}\right)=\left\{f \in \mathcal{M}\left(K_{g}\right) \mid f=0 \text { or }(f)+n \mathcal{D}_{P_{\infty}}+\mathcal{D}_{\underline{\hat{\mu}}\left(x_{0}\right)} \geq 0\right\}, \quad n \in \mathbb{N}_{0} .
$$

With $\phi(P, x), \psi\left(P, x, x_{0}\right)$ defined as in 3.15), 3.18) we obtain the following

Theorem B.1. Assume $\mathcal{D}_{\hat{\mu}\left(x_{0}\right)}$ to be nonspecial (i.e., $i\left(\mathcal{D}_{\hat{\mu}\left(x_{0}\right)}\right)=0$ ) and of degree $g \in \mathbb{N}$. For $n \in \mathbb{N}_{0}$, a basis for the vector space $\mathcal{L}\left(-n \mathcal{D}_{P_{\infty}}-\mathcal{D}_{\underline{\hat{\mu}}\left(x_{0}\right)}\right)$ is given by

$$
\begin{cases}\{1\}, & n=0 \\ \left\{\tilde{\pi}^{j}\right\}_{0 \leq j \leq(n-1) / 2} \cup\left\{\tilde{\pi}^{j} \phi\left(., x_{0}\right)\right\}_{0 \leq j \leq(n-1) / 2}, & n \text { odd } \\ \left\{\tilde{\pi}^{j}\right\}_{0 \leq j \leq n / 2} \cup\left\{\tilde{\pi}^{j} \phi\left(., x_{0}\right)\right\}_{0 \leq j \leq(n-2) / 2}, & n \text { even }\end{cases}
$$

or equivalently,

$$
\mathcal{L}\left(-n \mathcal{D}_{P_{\infty}}-\mathcal{D}_{\underline{\hat{\mu}}\left(x_{0}\right)}\right)=\operatorname{span}\left\{\left.\frac{\partial^{j}}{\partial x^{j}} \psi\left(., x, x_{0}\right)\right|_{x=x_{0}}\right\}_{0 \leq j \leq n}
$$

Proof. The elements in (B.3) easily seen to be linearly independent and belonging to $\mathcal{L}\left(-n \mathcal{D}_{P_{\infty}}-\right.$ $\left.\mathcal{D}_{\hat{\mu}\left(x_{0}\right)}\right)$. It remains to be shown that they are maximal. From $0=i\left(\mathcal{D}_{\hat{\mu}\left(x_{0}\right)}\right)=i\left(\mathcal{D}_{n P_{\infty}}+\right.$ $\left.\mathcal{D}_{\underline{\hat{\mu}}\left(x_{0}\right)}\right)$ and the Riemann-Roch theorem A.34 one obtains $r\left(-n \mathcal{D}_{P_{\infty}}-\mathcal{D}_{\underline{\hat{\mu}}\left(x_{0}\right)}\right)=n+1$ proving (B.3). In order to prove (B.4), one repeatedly uses the Schrödinger equation (3.21) to prove inductively that

$$
\begin{aligned}
& \frac{\partial^{2 m+2}}{\partial x^{2 m+2}} \psi\left(P, x, x_{0}\right)=(-\tilde{\pi})^{m+1}+R_{2 m+1}(P, x) \\
& \frac{\partial^{2 m+1}}{\partial x^{2 m+1}} \psi\left(P, x, x_{0}\right)=(-\tilde{\pi})^{m} \frac{\partial}{\partial x} \psi\left(P, x, x_{0}\right)+R_{2 m}(P, x),
\end{aligned}
$$

where $R_{n}\left(., x_{0}\right) \in \mathcal{L}\left(-n \mathcal{D}_{P_{\infty}}-\mathcal{D}_{\underline{\hat{\mu}}\left(x_{0}\right)}\right)$.

\section{ACKNOWLEDGMENTS}

F. G. would like to thank the organizers for their kind invitation to a most stimulating conference. 


\section{REFERENCES}

[1] S. I. Al'ber, Investigation of equations of Korteweg-de Vries type by the method of recurrence relations, J. London Math. Soc. (2) 19, 467-480 (1979) (Russian).

[2] S. I. Al'ber, On stationary problems for equations of Korteweg-de Vries type, Commun. Pure Appl. Math. 34, 259-272 (1981).

[3] N. Aronszajn and W. F. Donoghue, On exponential representations of analytic functions in the upper half-plane with positive imaginary part, J. Anal. Math. 5, 321-388 (1956-57).

[4] E. D. Belokolos, A. I. Bobenko, V. Z. Enol'skii, A. R. Its, and V. B. Matveev, "Algebro-Geometric Approach to Nonlinear Integrable Equations", Springer, Berlin, 1994.

[5] W. Bulla, F. Gesztesy, H. Holden, and G. Teschl, Algebro-geometric quasi-periodic finite-gap solutions of the Toda and Kac-van Moerbeke hierarchies, Mem. Amer. Math. Soc. 135-641, (1998).

[6] J. L. Burchnall and T. W. Chaundy, Commutative ordinary differential operators, Proc. London Math. Soc. (2), 21, 420-440 (1923).

[7] J. L. Burchnall and T. W. Chaundy, Commutative ordinary differential operators, Proc. Roy. Soc. London A118, 557-583 (1928).

[8] W. Craig, The trace formula for Schrödinger operators on the line, Commun. Math. Phys. 126, 379-407 (1989).

[9] L. A. Dickey, "Soliton Equations and Hamiltonian Systems", World Scientific, Singapore, 1991.

[10] B. A. Dubrovin, Periodic problems for the Korteweg-de Vries equation in the class of finite band potentials, Funct. Anal. Appl. 9, 215-223 (1975).

[11] H. M. Farkas and I. Kra, "Riemann Surfaces", 2nd ed., Springer, New York, 1992.

[12] J. D. Fay, "Theta Functions on Riemann Surfaces", Lecture Notes in Mathematics 352, Springer, Berlin, 1973.

[13] H. Flaschka, On the inverse problem for Hill's operator, Arch. Rat. Math. Anal. 59, 293-309 (1975).

[14] I. M. Gel'fand and L. A. Dikii, Asymptotic behaviour of the resolvent of Sturm-Liouville equations and the algebra of the Korteweg-de Vries equations, Russian Math. Surv. 30:5, 77-113 (1975).

[15] I. M. Gel'fand and L. A. Dikii, Integrable nonlinear equations and the Liouville theorem, Funct. Anal. Appl. 13, 6-15 (1979).

[16] F. Gesztesy, New trace formulas for Schrödinger operators, in "Evolution Equations", G. Ferreyra, G. R. Goldstein, and F. Neubrander (eds.), Marcel Dekker, New York, 1975, pp. 201-221.

[17] F. Gesztesy and H. Holden, Trace formulas and conservation laws for nonlinear evolution equations, Rev. Math. Phys. 6, 51-95 (1994).

[18] F. Gesztesy and H. Holden, On new trace formulae for Schrödinger operators, Acta Applicandae Math. 39, 315-333 (1995).

[19] F. Gesztesy and B. Simon, The xi function, Acta Math. 176 49-71 (1996).

[20] F. Gesztesy and R. Weikard, Spectral deformations and Soliton equations, in "Differential Equations with Applications to Mathematical Physics", W. F. Ames, E. M. Harrell II, and J. V. Herod (eds.), Academic Press, Boston, 1993, pp. 101-139.

[21] F. Gesztesy and R. Weikard, Picard potentials and Hill's equation on a Torus, Acta Math. 176, 73-107 (1996).

[22] F. Gesztesy, H. Holden, and B. Simon, Absolute summability of the trace relation for certain Schrödinger operators, Commun. Math. Phys. 168, 137-161 (1995).

[23] F. Gesztesy, H. Holden, B. Simon, and Z. Zhao, Trace formulae and inverse spectral theory for Schrödinger operators, Bull. Amer. Math. Soc. 29, 250-255 (1993).

[24] F. Gesztesy, H. Holden, B. Simon, and Z. Zhao, Higher order trace relations for Schrödinger operators, Rev. Math. Phys. 7, 893-922 (1995).

[25] H. Hochstadt, On the determination of a Hill's equation from its spectrum, Arch. Rat. Mech. Anal. 19, 353-362 (1965).

[26] A. R. Its and V. B. Matveev, Schrödinger operators with finite-gap spectrum and N-soliton solutions of the Korteweg-de Vries equation, Theoret. Math. Phys. 23, 343-355 (1975).

[27] C. G. T. Jacobi, Über eine neue Methode zur Integration der hyperelliptischen Differentialgleichungen und über die rationale Form ihrer vollständigen algebraischen Integralgleichungen, J. Reine Angew. Math. 32, 220-226 (1846). 
[28] S. Kotani and M. Krishna, Almost periodicity of some random potentials, J. Funct. Anal. 78, 390-405 (1988).

[29] A. Krazer, "Lehrbuch der Thetafunktionen", Chelsea, New York, 1970.

[30] M. G. Krein, Perturbation determinants and a formula for the traces of unitary and self-adjoint operators, Sov. Math. Dokl. 3, 707-710 (1962).

[31] B. M. Levitan, On the closure of the set of finite-zone potentials, Math. USSR Sbornik 51, 67-89 (1985).

[32] B. M. Levitan, "Inverse Sturm-Liouville Problems", VNU Science Press, Utrecht, 1987.

[33] H. P. McKean, Variation on a theme of Jacobi, Commun. Pure Appl. Math. 38, 669-678 (1985).

[34] H. P. McKean and P. van Moerbeke, The spectrum of Hill's equation, Invent. Math. 30, 217-274 (1975).

[35] H. P. McKean and E. Trubowitz, Hill's operator and hyperelliptic function theory in the presence of infinitely many branch points, Commun. Pure Appl. Math. 29, 143-226 (1976).

[36] D. Mumford, "Tata Lectures on Theta II", Birkhäuser, Boston, 1984.

[37] R. Narasimhan, "Compact Riemann Surfaces", Birkhäuser, Basel, 1992.

[38] S. Novikov, S. V. Manakov, L. P. Pitaevskii, and V. E. Zakharov, "Theory of Solitons", Consultants Bureau, New York, 1984.

[39] B. Simon, Spectral analysis of rank one perturbations and applications, in Mathematical quantum theory. II. Schrödinger operators, 109-149, CRM Proc. Lecture Notes, 8, Amer. Math. Soc., Providence, RI, 1995.

[40] E. Trubowitz, The inverse problem for periodic potentials, Commun. Pure Appl. Math. 30, 321-337 (1977).

Department of Mathematics, University of Missouri, Columbia, MO 65211

E-mail address: gesztesyf@missouri.edu

Department of Mathematics, University of Missouri, Columbia, MO 65211

Department of Mathematics, University of Missouri, Columbia, MO 65211

Current address: Institut für Mathematik, Strudlhofgasse 4, 1090 Wien, Austria

E-mail address: Gerald.Teschl@univie.ac.at 